\title{
An Idealized Numerical Study of Shear-Relative Low-Level Mean Flow on Tropical Cyclone Intensity and Size
}

\author{
Buo-Fu CHEN AND CHRistopher A. DAvis \\ National Center for Atmospheric Research, Boulder, Colorado \\ YING-HwA KUO \\ National Center for Atmospheric Research, and University Corporation for Atmospheric Research, Boulder, Colorado
}

(Manuscript received 19 October 2018, in final form 9 May 2019)

\begin{abstract}
Given comparable background vertical wind shear (VWS) magnitudes, the initially imposed shear-relative low-level mean flow (LMF) is hypothesized to modify the structure and convective features of a tropical cyclone (TC). This study uses idealized Weather Research and Forecasting Model simulations to examine TC structure and convection affected by various LMFs directed toward eight shear-relative orientations. The simulated TC affected by an initially imposed LMF directed toward downshear left yields an anomalously high intensification rate, while an upshear-right LMF yields a relatively high expansion rate. These two shearrelative LMF orientations affect the asymmetry of both surface fluxes and frictional inflow in the boundary layer and thus modify the TC convection. During the early development stage, the initially imposed downshear-left LMF promotes inner-core convection because of high boundary layer moisture fluxes into the inner core and is thus favorable for TC intensification because of large radial fluxes of azimuthal mean vorticity near the radius of maximum wind in the boundary layer. However, TCs affected by various LMFs may modify the near-TC VWS differently, making the intensity evolution afterward more complicated. The TC with a fast-established eyewall in response to the downshear-left LMF further reduces the near-TC VWS, maintaining a relatively high intensification rate. For the upshear-right LMF that leads to active and sustained rainbands in the downshear quadrants, TC size expansion is promoted by a positive radial flux of eddy vorticity near the radius of $34-\mathrm{kt}$ wind $\left(1 \mathrm{kt} \approx 0.51 \mathrm{~m} \mathrm{~s}^{-1}\right)$ because the vorticity associated with the rainbands is in phase with the storm-motion-relative inflow.
\end{abstract}

\section{Introduction}

A critical topic regarding tropical cyclone (TC) forecasting and disaster warning is the intensification and structural development of TCs. These complicated and nonlinear physical processes are to some degree related to environmental factors including ocean heat content (Lin et al. 2013), upper-level divergence (Elsberry and Jeffries 1996), and lower- to midtroposphere environmental relative humidity (Hill and Lackmann 2009; Dunion and Velden 2004). However, the effects of the environmental wind profile on a TC are particularly critical (DeMaria and Kaplan 1994, 1999; Emanuel et al. 2004; Kaplan et al. 2010; Zhang and Tao 2013; Chen et al. 2018). For example, the deep-layer vertical wind shear (VWS) magnitude from the environmental wind

\footnotetext{
Corresponding author: Buo-Fu Chen, bfchen777@gmail.com
}

profile has been found to have a strong relationship to TC development and its predictability (e.g., DeMaria and Kaplan 1999; Zhang and Tao 2013).

In a vertically sheared environment, TC intensification and structure development are influenced by the subsequent processes responding to the tilting vortex, such as undergoing vortex realignment and promoting convective asymmetry. The vortex realignment can be explained through the perspective of vortex Rossby wave (VRW) damping (Reasor and Montgomery 2001; Reasor et al. 2004). Specifically, perturbation of the vortex by VWS strongly projects onto a vertical and azimuthal wavenumber-1 VRW pattern, and their damping leads to a reduction of vortex tilt. TC (rapid) intensification often follows the vortex realignment after which the upper-level vortex precesses beyond $90^{\circ}$ to the left of the VWS vector (Jones 1995; Stevenson et al. 2014; Chen and Gopalakrishnan 2015). However, before 
the sheared TC becomes vertically realigned, the tilted structure promotes azimuthal asymmetry of convection and precipitation in the downshear quadrant (Corbosiero and Molinari 2002; Hence and Houze 2011; Reasor et al. 2013; DeHart et al. 2014). This convective asymmetry is considered as a negative factor for intensification because it transports lowentropy air by the downdrafts into the inflow boundary layer, and thus inhibits axisymmetric convection in the TC inner core (Powell 1990; Riemer et al. 2010, 2013; Riemer and Laliberté 2015).

While VWS generally limits TC intensification (e.g., DeMaria and Kaplan 1994, 1999; Hendricks et al. 2010), the predictability of TC structural development is still limited. Bhatia and Nolan (2013) documented that the intensity forecast for TCs under moderate VWS are characterized by larger errors. Recent observational studies (Nguyen et al. 2017; Rios-Berrios and Torn 2017; Fischer et al. 2018) showed that although the VWS typically forces a convective asymmetry within TCs, the magnitude and azimuthal distribution of this asymmetry can exhibit considerable variability, even among TCs affected by similar VWS magnitudes.

Several studies (Rappin and Nolan 2012; Finocchio et al. 2016; Onderlinde and Nolan 2014, 2016; Chen et al. 2018) have further linked the structural development of TCs affected by comparable VWS magnitudes to other properties associated with the vertically sheared background wind profile. Variability of TC development and formation arises from factors including the relative orientation of the surface or low-level wind to the VWS (Rappin and Nolan 2012; Chen et al. 2018), the height and depth of VWS (Finocchio et al. 2016), and the environmental helicity (Onderlinde and Nolan 2014, 2016).

The idealized simulations of Rappin and Nolan (2012) showed that whereas the surface wind orientation opposite to the VWS vector (i.e., upshear surface flow) favors TC formation and intensification, the surface wind orientation aligned with the VWS vector (i.e., downshear surface flow) inhibits TC formation. When a TC is affected by upshear-directed surface flow, superposition of this surface flow and the TC circulation contributes to a positive surface flux anomaly that is located in the left-of-shear quadrant and downwind of the downshear convective asymmetry. This positive surface flux anomaly helps recover the boundary layer equivalent potential temperature $\theta_{e}$ in the left-of-shear quadrant and then promotes convection in the upshear inner core. Subsequently, a vortex realignment of upperlevel circulation around the low-level TC center and TC intensification are likely to occur. In addition, Rappin and Nolan (2012, their Fig. 3) found that the difference of TC intensification due to distinct surface flow orientations is only present with a relatively warm sea surface temperature $\left(\mathrm{SST}>30.5^{\circ} \mathrm{C}\right.$ ) in their simulations. Note also that their results were based on idealized simulations, in which the ocean feedback is absent. This might be especially critical to the simulation with an upshear surface flow because the TC in this flow configuration has a relatively slow translation speed, which implies a relatively strong SST cooling beneath the TC center.

In the real atmosphere, an environmental wind profile characterized by northeasterly VWS and southwesterly monsoonal low-level flow (i.e., shear-relative flow directed toward upshear) favors long-lasting and active rainbands within western North Pacific TCs (Chen et al. 2014a,b, 2016). Chen et al. (2014a) also found correlations of these long-lasting rainbands to high TC expansion rate and less intensification. Our previous study (Chen et al. 2018) explored the simultaneous effect of VWS and low-level mean flow (LMF) on TC structure development for 180 western North Pacific TCs during 2003-16. Chen et al. (2018) showed that when a TC interacts with a LMF with left-of-shear orientation, the TC favors intensification over expansion. To the contrary, a LMF with right-of-shear or upshear orientations is favorable for size expansion. As suggested by a conceptual model based on composite analyses of satellite observations (Chen et al. 2018, their Fig. 14), TC structural evolution is attributed to the interaction between (i) the surface enthalpy flux associated with the surface wind asymmetry induced by the TC-LMF superposition and (ii) flow trajectories with respect to the moving TC center. Note that the storm-relative inflow trajectories are generally located on the downshear side regardless of the shear-relative LMF orientation. Therefore, for a TC affected by a left-ofshear LMF, the air mass influenced by high-enthalpy fluxes along the trajectory in the downshear quadrant is transported into the upshear inner core, promoting a relatively axisymmetric convection distribution and intensification.

Both Rappin and Nolan (2012) and Chen et al. (2018) have shown that LMF (or surface wind) orientation with respect to the VWS is an important factor affecting TC structural evolution. However, these studies disagree regarding a favorable flow configuration for intensification. A critical difference between the two studies is that Rappin and Nolan (2012) showed in an idealized modeling framework that an upshear LMF dynamically favors intensification, while Chen et al. (2018) showed from a statistical perspective that a left-of-shear LMF favors intensification in the western North Pacific. In addition, it is hard to directly compare the results 
between these two studies because (i) Chen et al. (2018) examined four orientations, while Rappin and Nolan (2012) examined only upshear and downshear orientations, and (ii) although Chen et al. (2018) had justified that the relationship between LMF orientation and TC development is not coincidently attributed to other environmental factors (e.g., VWS magnitude and environmental SST), this relationship is still affected by the ocean upwelling beneath the inner core, which is not considered in Rappin and Nolan (2012). Therefore, the current study utilizes idealized Weather Research and Forecasting (WRF) Model simulations to further explore the effect of eight shear-relative LMF orientations on TC structure evolution as well as the mesoscale processes that account for different convective features associated with distinct LMF orientations.

Section 2 describes the configurations of idealized WRF simulations and the calculation of an angular momentum budget to diagnose TC structural development. Preliminary examination of the structural development associated with various LMFs is presented in section 3, with an explanation for the discrepancy between Rappin and Nolan (2012) and Chen et al. (2018). Angular momentum budgets, which quantify the effects of distinct convective features on TC intensity and size, are presented in section 4 for TCs affected by LMFs with downshear-left and upshear-right orientations. Possible physical processes accounting for these distinct convective features associated with the two LMF orientations are addressed in section 5 . The concluding remarks are provided in section 6 .

\section{Methodology}

\section{a. Model configurations and experiment setups}

The Advanced WRF Model, version 3.4.1 (Skamarock et al. 2005), was used to conduct idealized simulations. These WRF simulations were fully compressible, Eulerian, and nonhydrostatic and used terrain-following hydrostatic pressure vertical coordinates with 35 levels ranging from the surface to $20 \mathrm{hPa}$. The integration period for each TC simulation was $120 \mathrm{~h}$. A larger geographically fixed outer domain measuring $7200 \mathrm{~km} \times 7200 \mathrm{~km}$ and an inner TCfollowing domain measuring $1440 \mathrm{~km} \times 1440 \mathrm{~km}$ were employed with two-way interaction. Grid dimensions for both domains were $360 \times 360$, with horizontal gridpoint spacings of 20 and $4 \mathrm{~km}$ for the outer and inner domains, respectively. The inner moving domain followed the 850-hPa vortex center.

The physics that parameterized convection on all grids utilized the new Thompson et al. (2008) microphysics scheme, which includes ice, snow, and graupel processes. To parameterize the surface fluxes, friction, and vertical mixing in the planetary boundary layer, the Yonsei University planetary boundary layer scheme (Hong et al. 2006) was used. Following previous idealized numerical studies regarding similar research topics (e.g., Onderlinde and Nolan 2014; Finocchio et al.2016), no cumulus convective parameterization or radiative scheme was employed. All of the simulations were performed on the ocean and on $f$ plane with a constant Coriolis torque equivalent to $15^{\circ} \mathrm{N}$ latitude. Furthermore, simulations with three different ocean feedback options were conducted: (i) a fixed SST of $29.5^{\circ} \mathrm{C}$, (ii) a higher fixed SST of $31.0^{\circ} \mathrm{C}$, and (iii) with ocean feedback included. For the experiments with ocean feedback, a simple 1D ocean mixed layer (OML) model (Davis et al. 2008) based on Pollard et al. (1972) was employed. This model simply requires specification of the initial OML depth $(30 \mathrm{~m})$, the temperature lapse rate $\left(0.14 \mathrm{~K} \mathrm{~m}^{-1}\right)$, and the wind stress at the surface.

This study aims to evaluate the sensitivity of TC development from an already TD-intensity dry vortex to an initial background flow maintained at the far-field domain boundary. The initial TC was a cloud-free, baroclinic vortex with a maximum wind of $18 \mathrm{~m} \mathrm{~s}^{-1}$ at a radius of $100 \mathrm{~km}$ and a height of $1.5 \mathrm{~km}$. The tangential wind $V_{\theta}$ distribution of this bogus vortex was similar to that of Chan and Chan (2014) and was given by

$$
V_{\theta}(r, z)=v_{\theta}(r) g(z)
$$

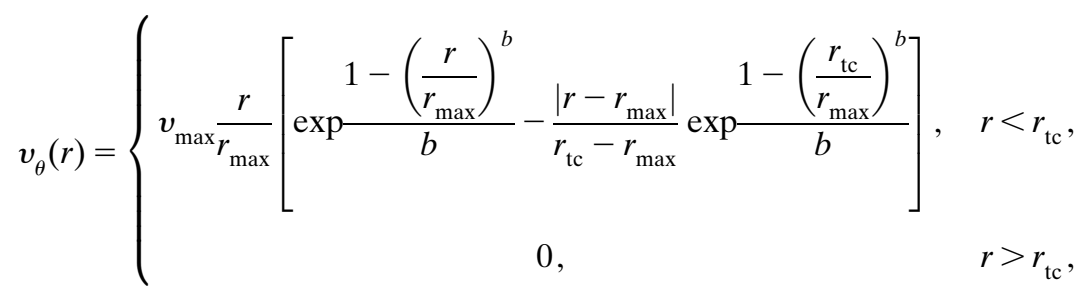


TABLE 1. Background flow velocities $\left(\mathrm{m} \mathrm{s}^{-1}\right)$ below $850 \mathrm{hPa}$ and above $200 \mathrm{hPa}$ for nine experiments with various LMF but equivalent VWS

\begin{tabular}{lll}
\hline \hline \multicolumn{1}{c}{ Experiment name } & $\begin{array}{c}\text { Wind below } \\
850 \mathrm{hPa}(u, v)\end{array}$ & $\begin{array}{c}\text { Wind above } \\
200 \mathrm{hPa}(u, v)\end{array}$ \\
\hline No LMF & $(0,0)$ & $(-10,0)$ \\
Right-of-shear LMF (Exp. RS) & $(0,6)$ & $(-10,6)$ \\
Upshear-right LMF (Exp. USR) & $(4.2,4.2)$ & $(-5.8,4.2)$ \\
Upshear LMF (Exp. US) & $(6,0)$ & $(-4,0)$ \\
Upshear-left LMF (Exp. USL) & $(4.2,-4.2)$ & $(-5.8,-4.2)$ \\
Left-of-shear LMF (Exp. LS) & $(0,-6)$ & $(-10,-6)$ \\
Downshear-left LMF (Exp. DSL) & $(-4.2,-4.2)$ & $(-14.2,-4.2)$ \\
Downshear LMF (Exp. DS) & $(-6,0)$ & $(-16,0)$ \\
Downshear-right LMF (Exp. DSR) & $(-4.2,4.2)$ & $(-14.2,4.2)$ \\
\hline
\end{tabular}

and

$$
g(z)=\left\{\begin{array}{cc}
\exp \left\{-1.7 \times\left[\frac{(z-1500)}{16000}\right]^{2}\right\}, & z>1500, \\
\exp \left\{-3.4 \times\left[\frac{(-z+1500)}{8000}\right]^{2}\right\}, & z<1500,
\end{array}\right.
$$

where $r$ is the radius from the vortex center, $z$ is the height, and $v_{\theta}(r)$ and $g(z)$ indicate the horizontal and vertical distribution of the tangential winds, the parameter $v_{\max }$ is the maximum tangential wind, $r_{\max }$ is the radius of the maximum wind, and $r_{\mathrm{tc}}$ is the zero-wind radius of the TC. In this study, the $v_{\max }, r_{\max }$, and $r_{\mathrm{tc}}$ are $18 \mathrm{~m} \mathrm{~s}^{-1}$ and 100 and $1800 \mathrm{~km}$, respectively. The shape parameter $b$ of the wind profile is 0.4 in this study. Although the initial dry vortex underwent substantial vertical tilting over the first few hours before convection developed and the boundary layer associated with a moist vortex was established, the structures of TCs at $6 \mathrm{~h}$ in our simulations with various LMF orientations were not substantially different because of the artificial and "dry" tilted vortex state.

To construct an environment in which the TC vortex was inserted, a prescribed background flow characterized by a unidirectional $10 \mathrm{~m} \mathrm{~s}^{-1}$ easterly VWS plus a $6 \mathrm{~m} \mathrm{~s}^{-1}$ mean flow directed toward each of the eight directional angles at every level was imposed in a quiescent, basic-state atmosphere. Thus, a set of control (CTL) experiments simulated TCs affected by the same VWS magnitude but nine distinct mean flows: eight of them were with distinct LMF orientations, and the other one was with no additional mean flow (Table 1). For each simulation, the initial wind below $850 \mathrm{hPa}$ and above $200 \mathrm{hPa}$ was vertically invariant, and the initial wind profile was linearly interpolated between these two heights. For example, Fig. 1 shows the initial wind vectors (a) D01 averaged flow for Exp. No LMF

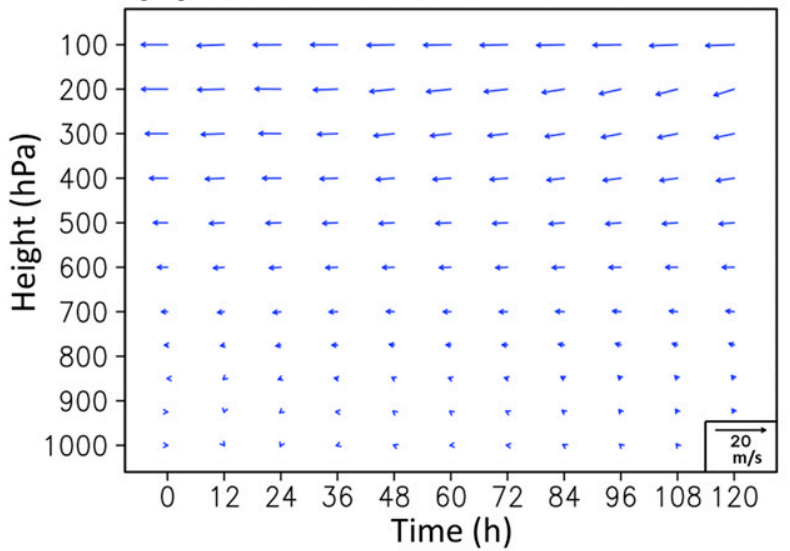

(b) D01 averaged flow for Exp. USR

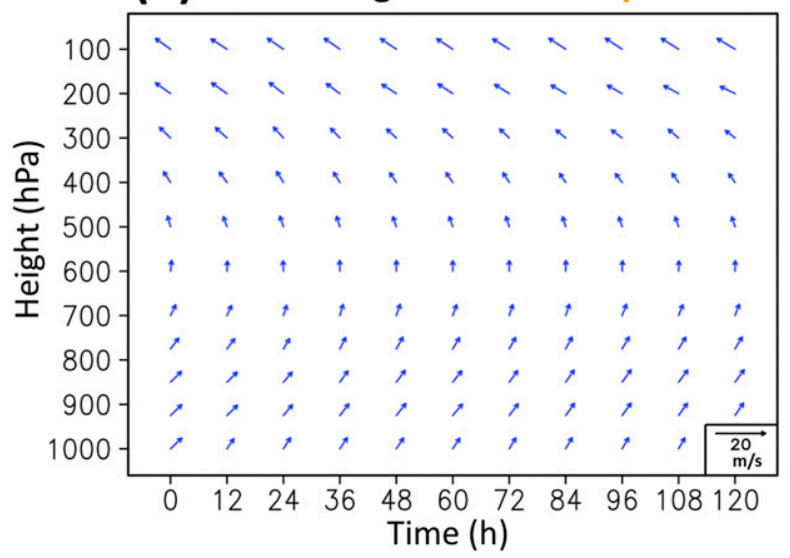

FIG. 1. Time-height diagram of domain-averaged wind vectors in the $7200 \mathrm{~km} \times 7200 \mathrm{~km}$ WRF outer domain for (a) Exp. No LMF and (b) Exp. USR.

from 1000 to $100 \mathrm{hPa}$ for the experiments with no shearrelative LMF (Fig. 1a, $t=0 \mathrm{~h}$ ) and a shear-relative LMF directed toward upshear right (Fig. $1 \mathrm{~b}, t=0 \mathrm{~h}$ ). Note that the magnitude, direction, depth, and height of the imposed VWS are equivalent among these experiments.

The geopotential height and temperature associated with the initial wind field (i.e., the background flow plus TC circulation) were explicitly determined by the thermal wind relation and were maintained by the far-field lateral boundary condition that is invariant in time. The calculation of the initial geopotential height, temperature, and moisture fields had to be with respect to a reference sounding profile, which was located $800 \mathrm{~km}$ east of the vortex center. This reference sounding was selected as the "moist tropical" sounding from Dunion (2011), to which was added $1^{\circ} \mathrm{C}$ at every level. In addition, a $29.5^{\circ} \mathrm{C} \mathrm{SST}$ was selected for the CTL simulations. Note that these parameters were determined according to additional sensitivity tests to various 
troposphere temperatures and SST (not shown). It is worth noting that we imposed the background flow as an initial condition and maintained it through the boundary conditions, rather than maintaining the background flow at each grid point by modifying the momentum equations (e.g., Rappin and Nolan 2012; Zhang and Tao 2013). Maintaining VWS at each grid point has the advantage of constant forcing of the vortex, while the downside to this approach is that, in reality, the vortex modifies the surrounding synoptic-scale flow. In our simulations, the way we impose the VWS and LMF allows the simulated TCs to modify the nearTC VWS differently in response to various LMFs, even though the domain-averaged VWS are generally stable as shown by the examples of experiment (Exp.) No LMF and Exp. USR in Fig. 1. In addition, the warm/ cold advection due to the prescribed background flow and the associated temperature gradient is present in our simulation, as it exists in the real atmosphere. Finally, two simulations with a third inner domain that has grid dimensions of $720 \times 720(480 \mathrm{~km} \times 480 \mathrm{~km})$ and spacing of $1.333 \mathrm{~km}$ were performed for upshearright and downshear-left LMF orientations, which are the two orientations focused in this paper. As in CTL simulations, the $1.333-\mathrm{km}$ simulations reproduce consistent TC structural evolution and development of convection (not shown), providing confidence in the interpretation of convection-related processes discussed in sections 3-5.

\section{b. Storm-relative absolute angular momentum budget}

A storm-relative absolute angular momentum (AAM) budget was applied for diagnosing the TC structural development. The AAM per unit mass of an air parcel about the rotation axis is defined as

$$
\mathrm{AAM}=v_{\theta} r+\frac{1}{2} f r^{2},
$$

where $v_{\theta}$ is the tangential velocity, $f$ the Coriolis parameter, and $r$ the radial distance from the TC center. The AAM approach is useful in interpreting the spinup mechanism of TC tangential wind within the boundary layer (Montgomery and Smith 2017, and reference therein). Namely, the increase of tangential velocity is strongly controlled by contributions from the inwardtransported AAM flux and the loss of AAM due to the friction. Although it has been assumed that the friction torque acts to reduce the tangential velocity within the boundary layer, the maximum tangential velocity in a TC has been found to occur in the inner-core boundary layer (Kepert 2006a,b; Montgomery et al. 2014; Sanger et al. 2014). The occurrence of this maximum tangential velocity is explained as follows: on one hand, as air parcels flow toward the center, they lose AAM to the surface due to friction. On the other hand, the corresponding tangential velocity $v_{\theta}$, may increase because of the decreased $r$. Therefore if the increase of $v_{\theta}$ due to the inward-transported AAM offsets the decrease of $v_{\theta}$ due to the loss of AAM, the maximum tangential velocity in the boundary layer can exceed the local value above the boundary layer (Montgomery and Smith 2017).

The storm-relative AAM budget in a moving cylindrical coordinate $(r, \theta, z)$ at a certain $r$ and model level can be obtained from the tangential equation of motion:

$$
\frac{d v_{\theta}}{d t}=\frac{-1}{\rho} \frac{\partial p}{r \partial \theta}-v_{r}\left(f+\frac{v_{\theta}}{r}\right)+F_{\theta},
$$

where $v_{\theta}$ and $v_{r}$ are the tangential and radial velocity components, $F_{\theta}$ is the frictional force in the tangential direction, $\rho$ the air density, and $p$ the pressure. Furthermore, the total derivative in Eq. (3a) in the coordinate following the storm motion, $\mathbf{C}=\left(c_{r}, c_{\theta}\right)$, is given by

$$
\frac{d}{d t}=\frac{\partial}{\partial t}+(\mathbf{V}-\mathbf{C}) \cdot \nabla .
$$

Multiplying Eq. (3a) by $r$ and some manipulations lead to

$$
\begin{aligned}
\frac{\partial \mathrm{AAM}}{\partial t} & +\left(v_{r}-c_{r}\right) \frac{\partial \mathrm{AAM}}{\partial r}+\left(v_{\theta}-c_{\theta}\right) \frac{\partial \mathrm{AAM}}{r \partial \theta}+w \frac{\partial \mathrm{AAM}}{\partial z} \\
& =\frac{-1}{\rho} \frac{\partial p}{\partial \theta}+r F_{\theta} .
\end{aligned}
$$

Then, by decomposing variables into azimuthal mean and asymmetric quantities and taking the azimuthal average of all terms, the local rate of change of azimuthally mean $\overline{\mathrm{AAM}}$ can be obtained:

$$
\begin{aligned}
\frac{\partial \overline{\mathrm{AAM}}}{\partial t}= & -r \overline{v_{r}} \bar{f}-r \bar{v}_{r} \bar{\zeta}-r \overline{\left(v_{r}-c_{r}\right)^{\prime} \zeta^{\prime}}-\bar{w} \frac{\partial \overline{\mathrm{AAM}}}{\partial z} \\
& -\overline{w^{\prime} \frac{\partial \mathrm{AAM}^{\prime}}{\partial z}-\frac{1 \partial p^{\prime}}{\rho^{\prime}}+r \overline{F_{\theta}}} .
\end{aligned}
$$

Note that the vertical component of relative vorticity for a generally axisymmetric TC is approximate as $\zeta=v_{\theta} / r+\partial v_{\theta} / \partial r$, and the asymmetric $f$ is zero on the $f$ plane.

Terms on the right-hand side in Eq. (5) are the contribution of the radial flux of planetary vorticity, the mean radial flux of relative vorticity, the eddy radial flux 


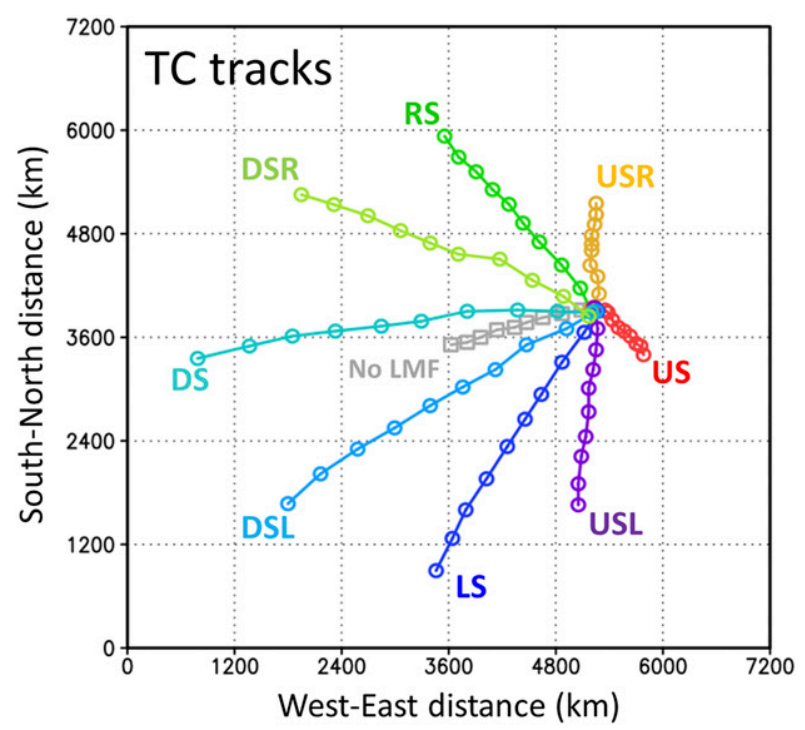

FIG. 2. TC tracks for nine CTL experiments in the $7200 \mathrm{~km} \times$ $7200 \mathrm{~km}$ outer domain. Experiments of TCs affected by different shear-relative LMF orientations (Table 1) are represented by different colors and texts. Circles and squares indicate 12-h intervals.

of relative vorticity, the vertical advection of AAM, the eddy vertical advection of AAM, eddy pressure gradient, and frictional torques to the local tendency of AAM. To a first approximation and as in previous studies (Merrill 1984; Cheung and Elsberry 2002; Smith et al. 2011; Chan and Chan 2014), the current study mainly investigated the role of the mean radial flux of relative vorticity and the eddy radial flux of relative vorticity in Eq. (5) because the vertical AAM advection and eddy pressure gradient terms are comparably small and thus negligible. Note that although friction plays an important role in the boundary, it is always a negative contribution.

\section{TC structural evolution affected by various shear-relative LMF orientations}

\section{a. Intensity and size evolution for the CTL simulations}

The tracks of the nine CTL TCs (Table 1) that are all affected by $10 \mathrm{~m} \mathrm{~s}^{-1}$ easterly VWS but with various LMFs are examined (Fig. 2). The TC in Exp. No LMF (Fig. 2, gray track) moves west with a speed of approximately $4.1 \mathrm{~m} \mathrm{~s}^{-1}$ and exhibits a $1.0 \mathrm{~m} \mathrm{~s}^{-1}$ southward drift. This drift is attributed to the interaction between the cyclonical low-level TC vortex and the anticyclone circulation above the TC (Wu and Emanuel 1993). For the other eight TCs affected by the LMFs, the translation velocity is attributed to the $10 \mathrm{~m} \mathrm{~s}^{-1}$ easterly VWS flow plus the steering effect caused by the $6 \mathrm{~m} \mathrm{~s}^{-1}$ background flow. For example, the TC zonal translation velocity in Exp. RS (Fig. 2, green track) is approximately equivalent to the TC translation velocity in Exp. No LMF plus a $6 \mathrm{~m} \mathrm{~s}^{-1}$ northward motion. Furthermore, the steady TC motions as shown in Fig. 2 suggest that the prescribed flow configurations generally hold through the simulations (see also Fig. 1).

The intensity $\left(V_{\max }\right)$ defined as the maximum $10-\mathrm{m}$ wind speed and size defined as the azimuthally averaged maximum radial extent of the $34-\mathrm{kt}$ winds $(\mathrm{R} 34 ; 1 \mathrm{kt} \approx$ $0.51 \mathrm{~m} \mathrm{~s}^{-1}$ ) of the nine CTL TCs with a fixed SST of $29.5^{\circ} \mathrm{C}$ are examined (Figs. 3a,c). Whereas TCs in Exp. RS (Fig. 3a, green) and Exp. USR (Fig. 3a, gold) have slower onsets of intensification and relatively low intensification rates, the TCs in Exp. LS (Fig. 3a, blue) and Exp. DSL (Fig. 3a, light blue) have relatively large intensification rates. Both intensification and expansion are simultaneously examined with a $V_{\max }-\mathrm{R} 34$ diagram (Fig. 3c) during 12-60 h (Fig. 3a, black vertical lines). The TC affected by an upshear-right LMF (Fig. 3c, gold line) favors expansion and increases its size to about $170 \mathrm{~km}$ at $60 \mathrm{~h}$, with relatively weak intensity. While TCs affected by downshear-left, downshear, and downshearright LMFs have relatively high intensification rates relative to low expansion rates. Although these TCs reach nearly the same intensity later in the simulations (Fig. 3a, after $84 \mathrm{~h}$ ), these simulations are generally consistent with the observational analyses of Chen et al. (2018) regarding the relationship between shear-relative LMF orientation and the pathways of TC structural development.

\section{b. Testing a higher SST and the influence of ocean feedback}

Recalling that the results of idealized simulations of Rappin and Nolan (2012) and observational analyses of Chen et al. (2018) disagree regarding a favorable flow configuration for intensification, this subsection presents the sensitivity tests of using a higher SST of $31^{\circ} \mathrm{C}$ and including ocean feedback to explore possible factors in the model that may explain the discrepancy between the two studies.

For the CTL simulations, a fixed SST of $29.5^{\circ} \mathrm{C}$ was used in the simulation to reach consistent results with the observational statistics (section 3a). However, if the fixed SST is increased to $31^{\circ} \mathrm{C}$, TC structural evolutions in response to various LMFs become more complicated (Figs. 3b,d). Although both intensity evolutions (Figs. 3a,b) and $V_{\max }-\mathrm{R} 34$ diagrams (Figs. 3c,d) before $30 \mathrm{~h}$ are consistent for the two SST setups, the intensification rates of TCs affected by upshear-left, left-ofshear, downshear-left, and downshear LMFs (Fig. 3b, cold colored lines) become small after about $30 \mathrm{~h}$. In this 
(a) Intensity, SST $=29.5^{\circ} \mathrm{C}$

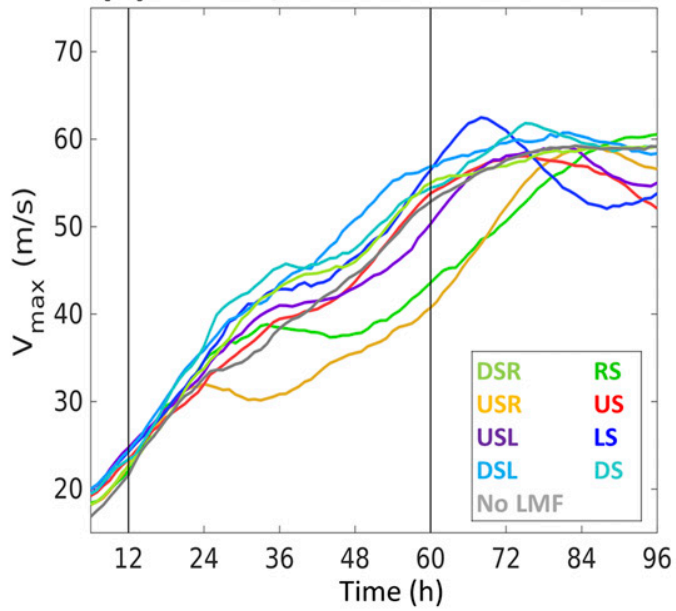

(c) V-R diagram, SST $=29.5^{\circ} \mathrm{C}$

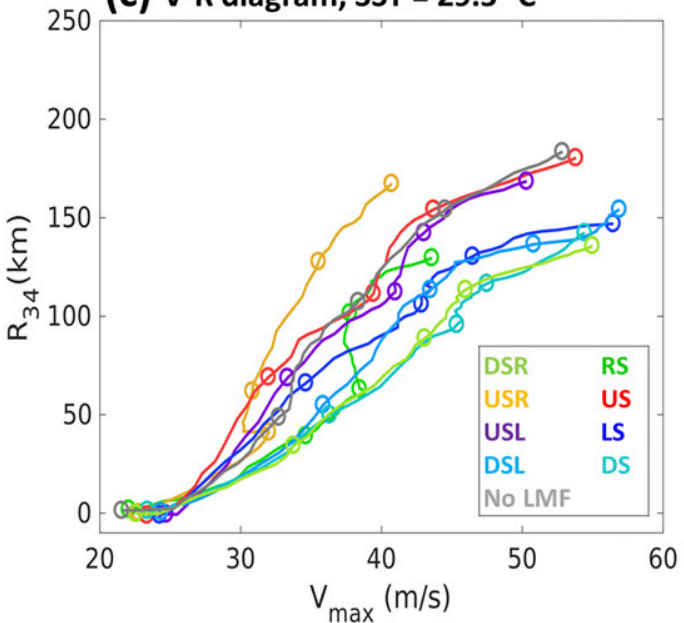

(b) Intensity, SST $=31^{\circ} \mathrm{C}$

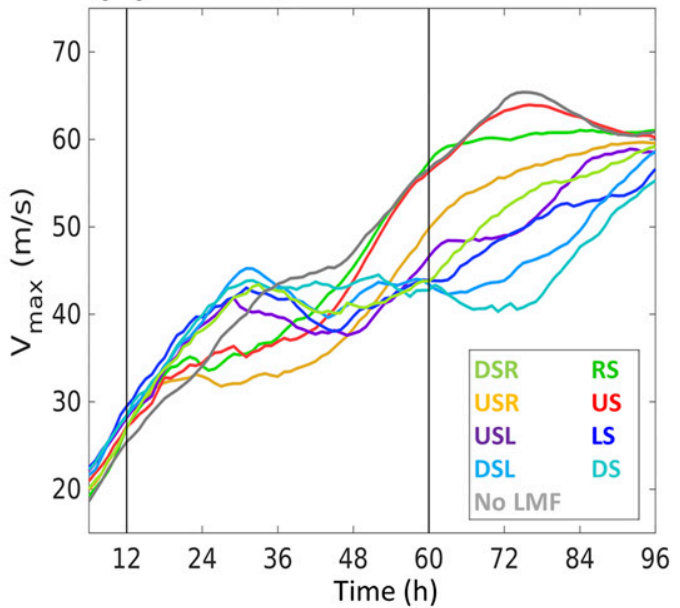

(d) V-R diagram, SST $=31^{\circ} \mathrm{C}$

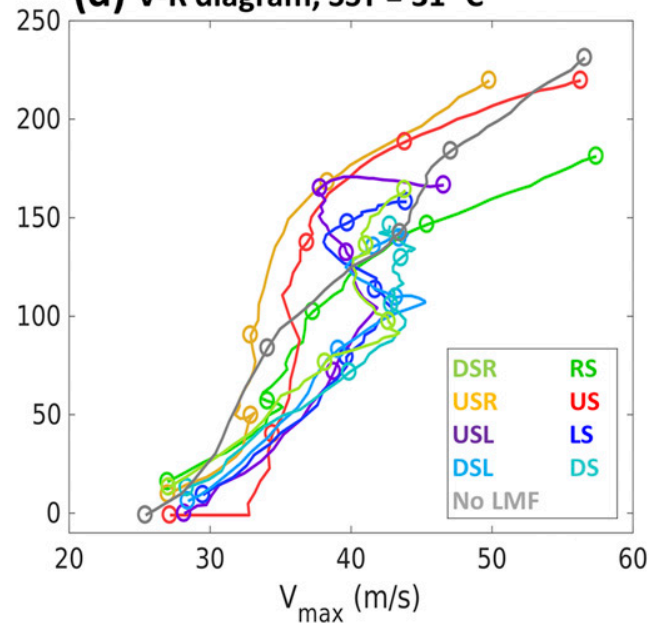

FIG. 3. (a) TC intensity evolution and (c) intensity-size diagram during 12-60 h for the nine CTL experiments $\left(\mathrm{SST}=29.5^{\circ} \mathrm{C}\right)$. The circles in (c) indicate 12-h intervals. (b), (d) As in (a) and (c), but for the sensitivity tests with a higher SST of $31^{\circ} \mathrm{C}$.

manner, the experiments with $31^{\circ} \mathrm{C}$ SST lead to a consistent result with Rappin and Nolan (2012) that an upshear LMF is related to a higher intensification rate while a downshear LMF is related to a lower intensification rate. It is worth noting that TCs affected by an upshear-right LMF have the highest expansion rates with respect to intensification rate for both SST setups (Figs. 3c,d, gold lines).

The deceleration of intensification rates for Exp. USL to Exp. DS (Fig. 3b, cold-colored lines) could be explained as follows. The TCs in the higher-SST experiments generally have larger rainfall extents and more active rainbands outside the inner core (not shown) than the CTL experiment with a $29.5^{\circ} \mathrm{C} \mathrm{SST}$. This explains the generally large TC size in Fig. 3d compared to Fig. 3c. Moreover, this may imply that boundary layer recovery in the left-of-shear quadrant from the low $-\theta_{e}$ air due to the downshear convection is critical in higher-SST experiments, which is the physical process emphasized in Rappin and Nolan (2012). Thus, the deceleration of intensification rates for Exp. USL to Exp. DS may be attributed to the intrusion of rainband-induced low- $\theta_{e}$ air and an insufficient $\theta_{e}$ recovery within the left-of-shear boundary layer.

As TCs affected by different shear-relative LMFs have various storm translation speeds (Fig. 1), the effect of ocean feedbacks on TC structural evolution is explored when examining the role of the shear-relative LMFs. Previous studies (Schade and Emanuel 1999; Lin et al. 2003; Dare and McBride 2011) have shown that the cooling of SST due to a TC depends on intensity, translation speed, and the OML depth. The magnitude of the SST cooling can be up to $9^{\circ} \mathrm{C}$ in an extreme case of 
Typhoon Kai-Tak in 2000 as documented by Lin et al. (2003). Therefore, a set of experiments was conducted to examine the effect of LMF orientation on a TC when the ocean feedback is included.

The evolutions of inner-core SST (average within 200-km radius) for TCs in the OML experiments are examined in Fig. 4. Although all TCs are associated with SST cooling over $1.5 \mathrm{~K}$, the SST cooling is most pronounced for TCs affected by upshear and upshearright LMFs due to their slowest translation speeds (Fig. 1). The upshear simulations (Fig. 4a, red line) have SST cooling of about $4 \mathrm{~K}$ in the TC inner core. Compared to the CTL simulations (Fig. 3), the upshear and upshear-right simulations exhibit slower intensification rates and reach a lower maximum intensity because of the decreased SST (Fig. 4b, red and gold lines). The TC affected by an upshear-right LMF also favors expansion most relative to intensification (Fig. 4c, gold lines). For the right-of-shear case (Fig. 4, green line), the TC could not intensify anymore after $48 \mathrm{~h}$ (Fig. 4b) because of very asymmetric convection (not shown), weak TC circulation (not shown), SST reduction over $1 \mathrm{~K}$ (Fig. 4a), and the imposed $10 \mathrm{~m} \mathrm{~s}^{-1}$ VWS. On the other hand, TCs affected by LMFs with upshear-left, left-of-shear, to downshear orientations still have relatively high intensification rates and maximum intensities (Fig. 4b, lines with cold colors). These results suggest that the inclusion of ocean feedbacks leads to weaker maximum intensity for TCs affected by upshear LMFs, but the relationship between the shear-relative LMF and TC structural evolution is consistent between the OML experiments (Fig. 4c) and the CTL simulations (Fig. 3c).

Based on the experiments of the higher, $31^{\circ} \mathrm{C} \mathrm{SST}$ and the inclusion of ocean feedback, it is suggested that TC intensification in response to various LMF orientations is sensitive to the thermodynamic conditions provided, except for the early development stage before $36 \mathrm{~h}$. During the first 36-h simulation, TCs affected by downshear-left LMF have high intensification rates. However, TC intensity evolution in different experiment sets exhibits variability after $36 \mathrm{~h}$ presumably because of the relatively effective boundary layer $\theta_{e}$ recovery when a higher SST is used and the effect of SST cooling beneath the TC. These thermodynamic processes may explain the discrepancies between observational analyses (Chen et al. 2018) and results from Rappin and Nolan (2012), who focused on the kinematic response to distinct shearrelative surface wind directions when a fixed $30.5^{\circ} \mathrm{C}$ SST is provided. In addition, because TCs affected by upshear-right LMFs have consistently high expansion rates relative to intensification in the three experiment sets (Fig. 3c for CTL, Fig. 3d for higher
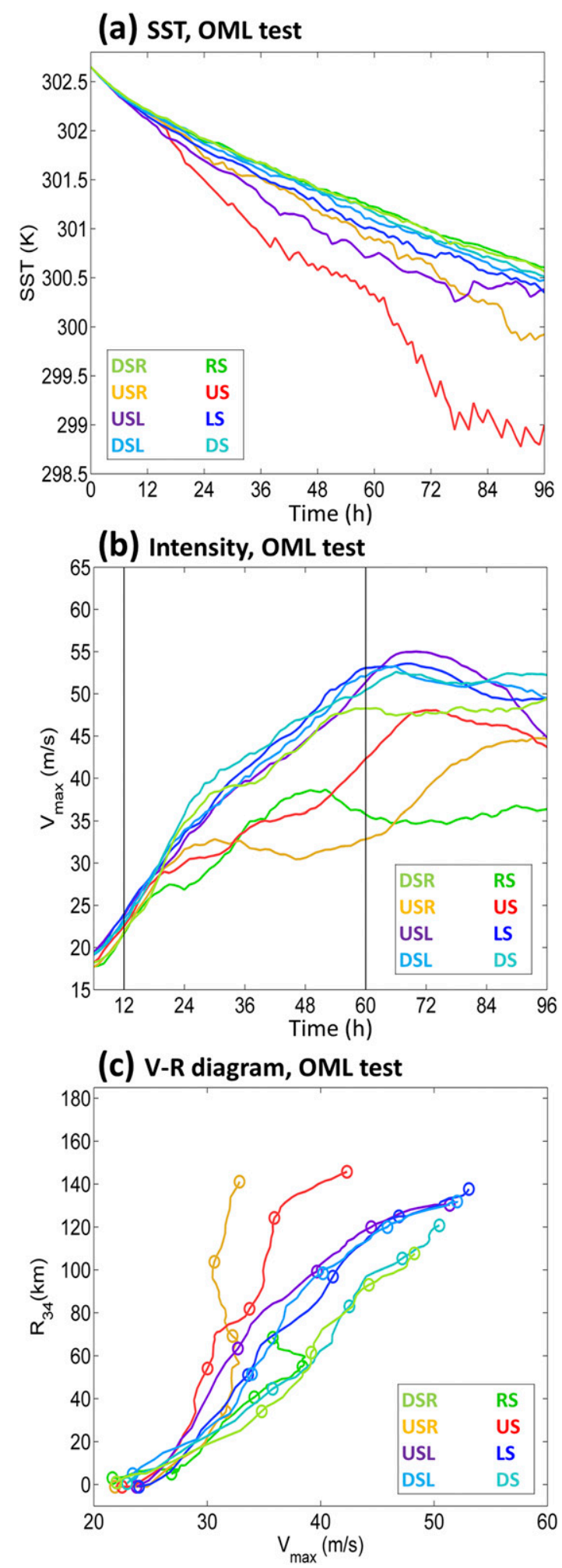

FIG. 4. (a) Evolutions of mean TC inner-core (0-200 km) sea surface temperature, (b) TC intensity evolutions, and (c) intensitysize diagrams during $12-60 \mathrm{~h}$ for TCs in experiments including ocean feedback. The circles in (c) indicate 12-h intervals. 

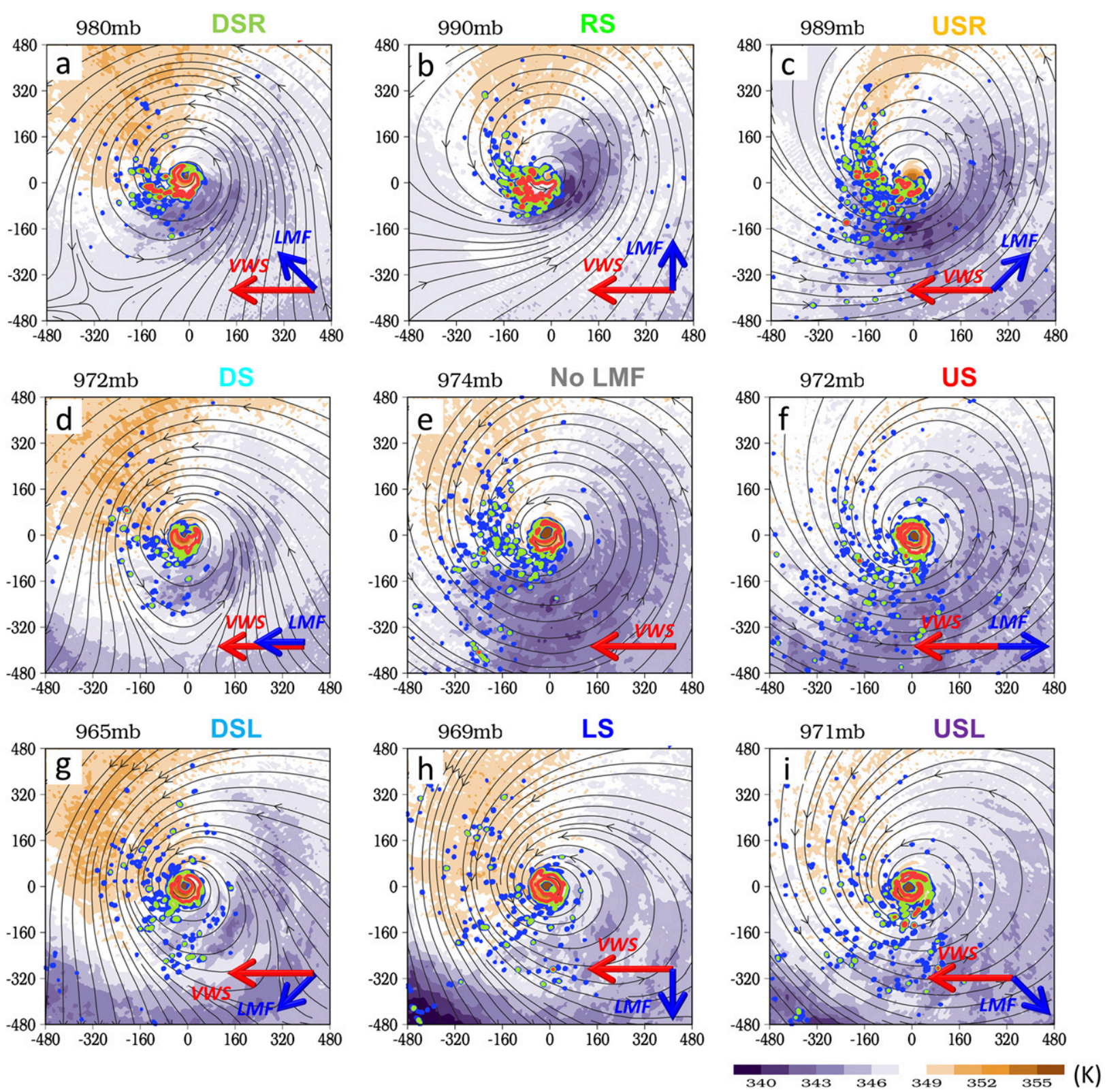

FIG. 5. Selected 30-54-h-averaged fields for nine CTL experiments: $0-1-\mathrm{km}$ streamlines (the spacing between streamlines is proportional to winds speed), $0-1-\mathrm{km}$ mixing ratio of rain (contoured at $0.5,0.7$, and $1.1 \mathrm{~g} \mathrm{~kg}^{-1}$ with blue, green, and red contours), and $0-$ $1-\mathrm{km}$ equivalent potential temperature (shaded according to the color bar at the lower right). The 24-h-averaged MSLP is labeled at the upper left of each panel. Schematic of the prescribed VWS and LMF for each experiment is shown at the lower left of each panel. The axes are distances $(\mathrm{km})$ from the TC center.

SST, and Fig. 4c for ocean feedback), the rest of this paper subsequently focuses on the physical processes accounting for the TC structural evolution in Exp. USR and compares the results with that based on Exp. DSL, which has an opposite LMF orientation. Note that the analyses for Exp. DSL are focused on the early development stage, during which consistent results could be obtained with different thermodynamic setups, and the TC has not yet substantially modified the near-TC VWS that also affects intensification (discussed later in section 5).

\section{c. Asymmetric features induced by shear-relative $L M F$ S}

The asymmetric features during $30-54 \mathrm{~h}$ of wind, rain, and $\theta_{e}$ are examined for the CTL TCs affected by various shear-relative LMFs (Fig. 5). Consistent with previous studies (e.g., Riemer et al. 2010; Reasor et al. 2013), the 

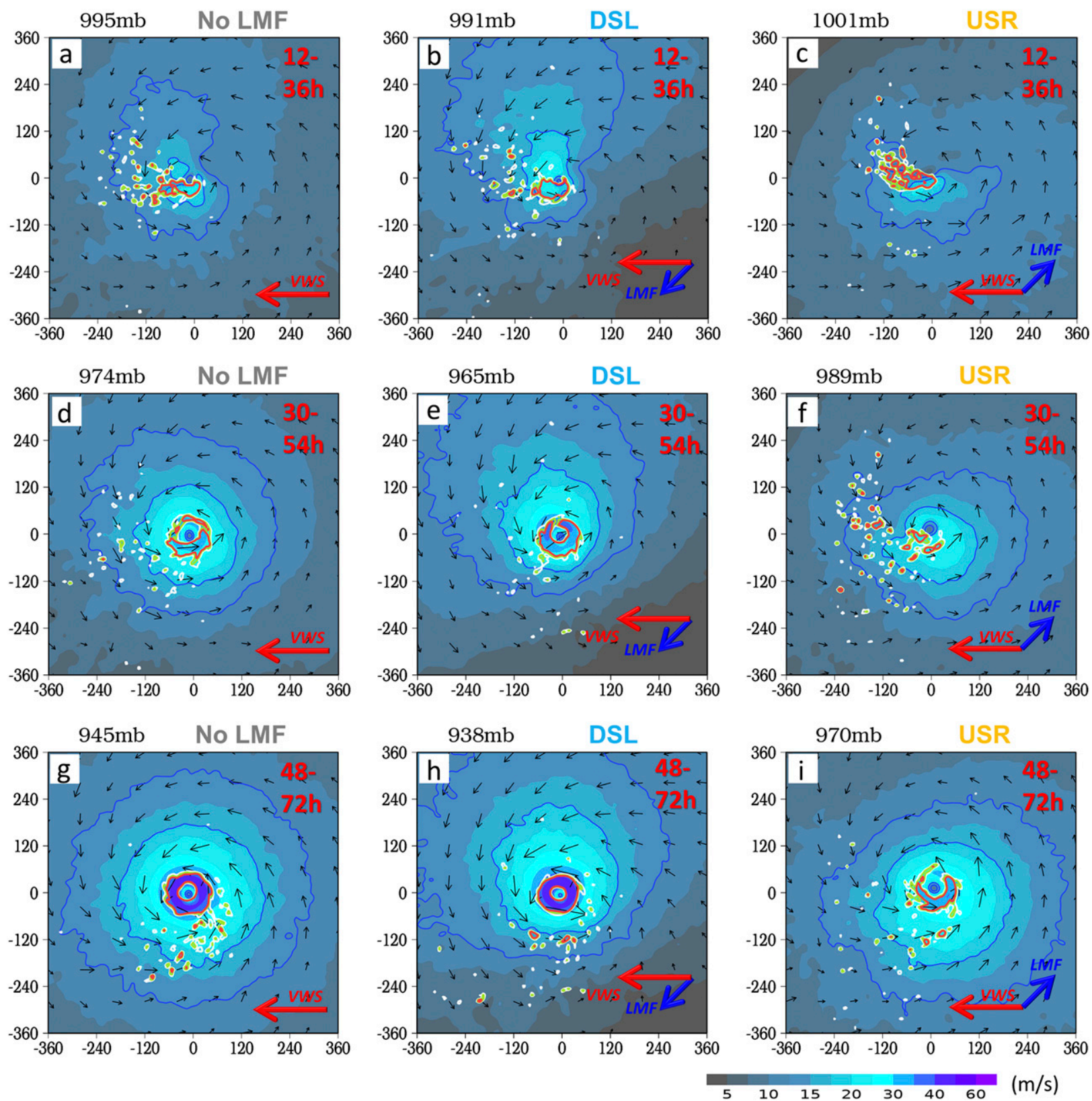

FIG. 6. (top) Selected 12-36-h-averaged fields for TCs affected by (a) no LMF, (b) downshear-left LMF, and (c) upshear-right LMF. These fields include surface wind (vectors, with shading according to the color bar at the lower right) and the mixing ratio of rain within $0-1 \mathrm{~km}$ (contoured at $0.7,0.9$, and $1.1 \mathrm{~g} \mathrm{~kg}^{-1}$ with white, green, and red contours). The blue contours indicate the surface wind speeds of 34 and $25 \mathrm{kt}$. (middle),(bottom) As in (a)-(c), but for during (d)-(f) 30-54 and (g)-(i) 48-72 h. The 24-h-averaged MSLP is labeled at the upper left of each panel. Schematics of VWS and LMF are shown in the lower right. The axes are distances $(\mathrm{km})$ from the TC center.

TC-VWS interaction in the no-LMF simulation (Fig. 5e) contributes to a downshear-left rainfall asymmetry (Fig. 5e, contours), which is associated with a moist envelope (Fig. 5e, warm-shaded colors) extending to the downshear-right quadrant. However, the simulations with distinct shear-relative LMF orientations (Fig. 5, except for Fig. 5e) show considerable differences in the $\theta_{e}$ and rainfall distributions. Whereas Exp. USR
(Fig. 5c) exhibits relatively active rainbands in the downshear quadrants, Exp. DSL (Fig. 5g) exhibits relatively symmetric inner-core convection. Exp. DSL also exhibits substantial high- $\theta_{e}$ air in the downshear-right quadrant (Fig. 5g, warm-shaded colors) while Exp. USR exhibits a relatively narrow moist envelope (Fig. 5c, warm-shaded colors). The amount of high- $\theta_{e}$ air $\left(\theta_{e}>\right.$ $349 \mathrm{~K}$ in Fig. 5) in the downshear-right quadrant could 
be attributed to the surface latent heat flux, which is proportional to the ground-relative surface wind speed in the TC outer region (Fig. 6, contours). Besides, the distribution of low- $\theta_{e}$ air (Fig. 5, cold-shaded colors) appears to be associated with the rainbands. Moreover, greater amount of low $\theta_{e}$ air near the inner core yields slower rates of intensification [Figs. 5a-c; the minimum sea level pressures (MSLP)].

Recalling that the TC affected by an upshear-right LMF favors expansion over intensification (Fig. 2, gold line), the surface wind and the rainfall at $12-36,30-54$, and $48-72 \mathrm{~h}$ are further examined for the TCs in Exp. USR, with comparisons to Exp. No LMF, and Exp. DSL. At 12-36h (Figs. 6b,c), enhanced positive surface wind anomalies are present in the right semicircles with respect to the LMF vector. The TC in Exp. USR also has larger asymmetric rain rates than the TC in Exp. DSL. At 30-54h (Figs. 6e,f), Exp. DSL exhibits rainfall associated with organized eyewall cloud and an MSLP of $965 \mathrm{hPa}$ (Fig. 6e), while Exp. USR has active rainbands occurring in the downshear quadrants (Fig. 6f) and later development of eyewall convection at 54-78 h (Fig. 6i). Note that Exp. USR has a TC with much stronger outer wind and larger size (Fig. 6i; see also Fig. 2b: $V_{\max }=$ $40 \mathrm{~m} \mathrm{~s}^{-1}$ and R34 $=170 \mathrm{~km}$ at $60 \mathrm{~h}$ for Exp. USR) than the TC in Exp. DSL at comparable intensities (Fig. 6e; see also Fig. 2b: $V_{\max }=40 \mathrm{~m} \mathrm{~s}^{-1}$ and $\mathrm{R} 34=85 \mathrm{~km}$ at $30 \mathrm{~h}$ for Exp. DSL). As the different convective features are likely to account for the distinct structure evolution for the TCs in Exp. DSL (Figs. 6b,e,h) and Exp. USR (Figs. 6c,f,i), the next section aims to quantify the feedback from these convective features to intensity and size based on the storm-relative AAM budget described in section 2b. Furthermore, as it is not easy to link the $\theta_{e}$ anomaly with the asymmetric rainfall magnitudes (Figs. 5c,g), extended analyses are shown later in section 5 to reveal the processes leading to the distinct convective features in Exp. DSL and Exp. USR.

\section{AAM budgets quantifying TC intensification and expansion}

The TC in Exp. DSL begins to develop at about $24 \mathrm{~h}$, and the eyewall is established at about $30 \mathrm{~h}$ (Figs. 7a,b, 40-dBZ contour). The azimuthally averaged tangential wind at the radius of maximum wind (RMW) increases by $20 \mathrm{~m} \mathrm{~s}^{-1}$ from 24 to $42 \mathrm{~h}$ (Fig. $8 \mathrm{a}$, blue dotted line). It is suggested that the development of the eyewall convection mutually promotes the increase of the radial inflow within the inner core (Fig. $7 \mathrm{~b}, r<100 \mathrm{~km}$ ). On the other hand, the TC in Exp. USR begins to develop at about $36 \mathrm{~h}$ with a relatively low intensification rate (Figs. 7c,d, shaded colors, and Fig. 8b, blue dotted line).
An organized eyewall is not present until $60 \mathrm{~h}$ (Figs. 7c,d, 40-dBZ contour), and the inner-core radial inflow in Exp. USR is weaker than that in Exp. DSL (Fig. 7d). However, a relatively high expansion rate is associated with Exp. USR (comparing the black lines in Figs. 8a and $8 b)$.

To quantify the effect on TC intensification of the increasing radial inflow in Exp. DSL (Figs. 7a, 7b, and $8 \mathrm{a}$ ), the AAM tendency, mean radial flux of vorticity, and eddy radial flux of vorticity at the RMW are examined (Fig. 8c). Consistent with previous studies (e.g., Merrill 1984; Cheung and Elsberry 2002; Chan and Chan 2014), the mean radial vorticity flux (Fig. 8c, thin dashed blue line) is the dominant term for TC intensification. From 24 to $42 \mathrm{~h}$, the time-averaged mean radial vorticity flux of approximately $75 \mathrm{~m}^{2} \mathrm{~s}^{-2}$ is about 4 times larger than the averaged eddy radial vorticity flux of approximately $18 \mathrm{~m}^{2} \mathrm{~s}^{-2}$ (Fig. 8c, thin solid blue lines). Moreover, the total radial flux of vorticity (Fig. 8c, thick blue lines) in Exp. DSL is about 2 times larger than that in Exp. USR (Fig. 8d, thick blue lines). In addition, the time-radius Hovmöller diagrams of the AAM budgets show that, for Exp. DSL (Figs. 9a-c), the mean radial flux of vorticity near the RMW during the first $12 \mathrm{~h}$ of intensification (Fig. 9b, pink box) is larger than that in Exp. USR (Fig. 9e, pink box). It is suggested that the strong radial inflow and the early developed eyewall in Exp. DSL (Figs. 6e and 7b, contours) contribute to larger mean radial vorticity flux in the inner core, in turn, leading to relatively high intensification rates.

As the TC in Exp. USR exhibits active rainbands and a relatively asymmetric convective feature in the downshear quadrants (section $3 \mathrm{c}$ ), this section also aims to quantify the effect of these asymmetric convective features on TC expansion. As shown in Figs. 9c and 9f, the negative eddy radial flux of vorticity is present outside of the inner core and near R34 in Exp. DSL (Fig. 9c, pink box), while a positive contribution is present near R34 in Exp. USR (Fig. 9f, pink box). Furthermore, unlike intensification (Figs. 8c,d), the eddy radial flux of vorticity (Figs. 8e,f, thin solid red lines) plays a critical role in TC expansion. For Exp. USR, the contribution of eddy radial vorticity flux near the R34 (Fig. 8f, thin solid line) is about one-half of the mean radial vorticity flux (Fig. 8f, thin dashed line). In contrast, the negative eddy radial vorticity flux in Exp. DSL during 24-42 h (Fig. 8e, thin solid line) offsets the mean radial vorticity flux. Therefore, the positive eddy radial vorticity flux near the R34 is important for determining TC size expansion rates.

To understand the effect of active downshear-left rainbands in Exp. USR (Fig. 10b, contour) on TC size expansion, the eddy radial vorticity flux and the radial 
(a) 0-1-km v $\left(\mathrm{m} \mathrm{s}^{-1}\right)$

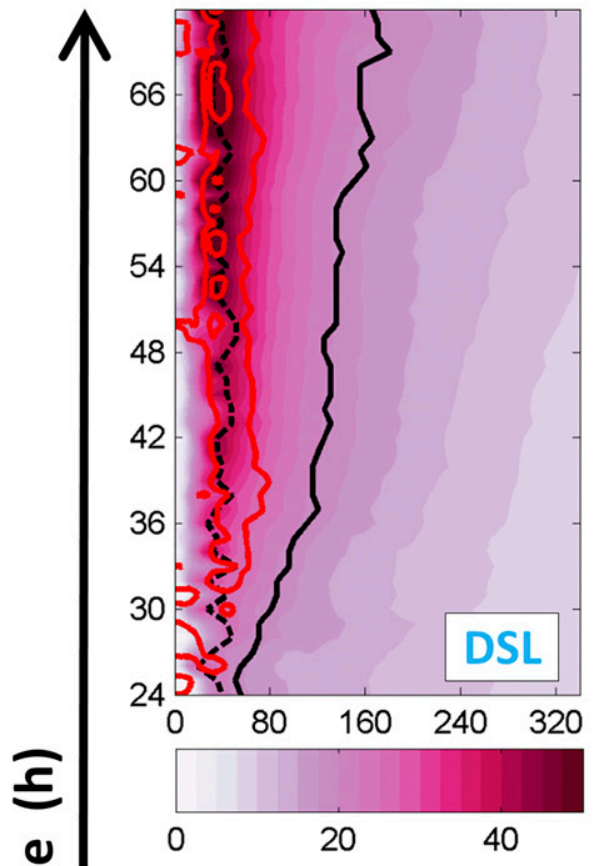

(c) 0-1-km v $\left.\mathrm{t}_{\mathrm{t}} \mathrm{m} \mathrm{s}^{-1}\right)$

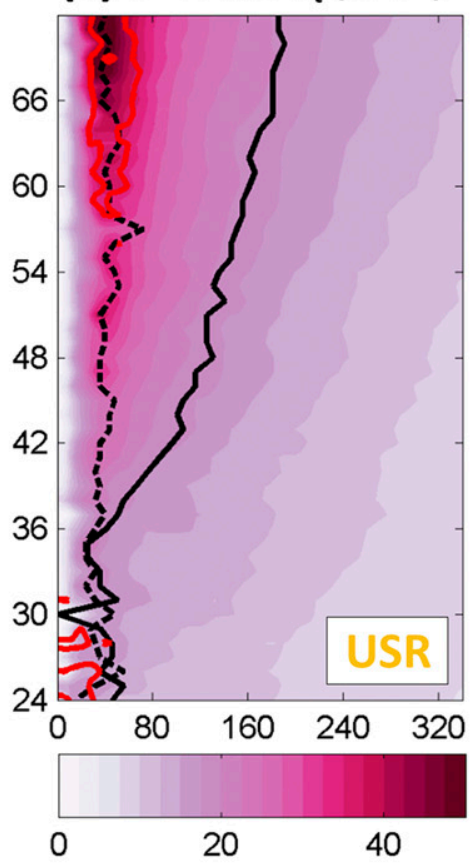

(b) 0-1-km V $\mathrm{r}_{\mathrm{r}}\left(\mathrm{m} \mathrm{s}^{-1}\right)$

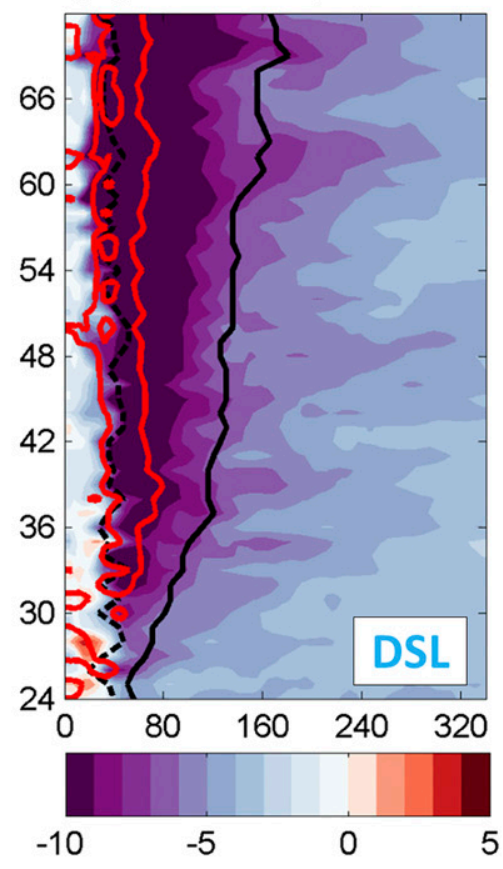

(d) 0-1-km $\mathrm{V}_{\mathrm{r}}\left(\mathrm{m} \mathrm{s}^{-1}\right)$

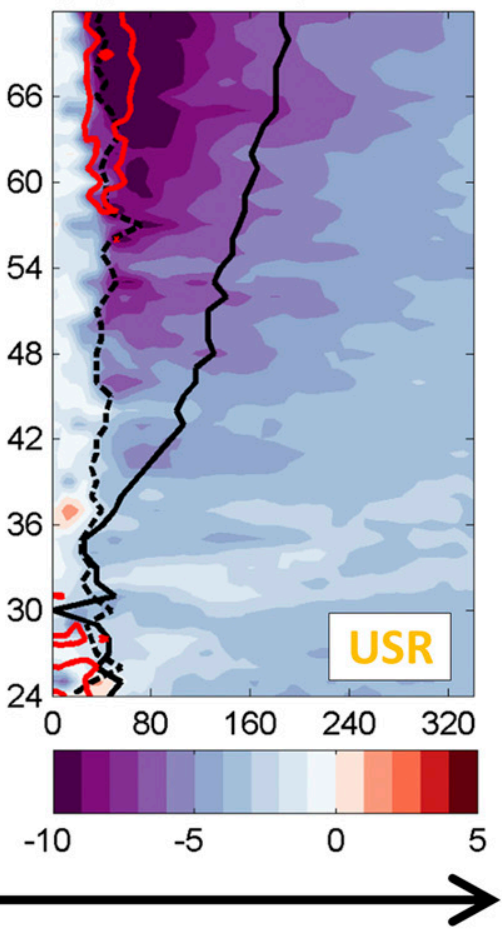

Radius (km)

FIG. 7. Time-radius Hovmöller diagrams of azimuthally averaged (a),(c) 0-1-km tangential wind and (b),(d) 0-1-km radial wind for the TCs in (a),(b) Exp. DSL and (c),(d) Exp. USR. Red contours indicate the maximum reflectivity of 40 and $50 \mathrm{dBZ}$. Black dotted line and black solid line indicate the RMW and R34, respectively. 

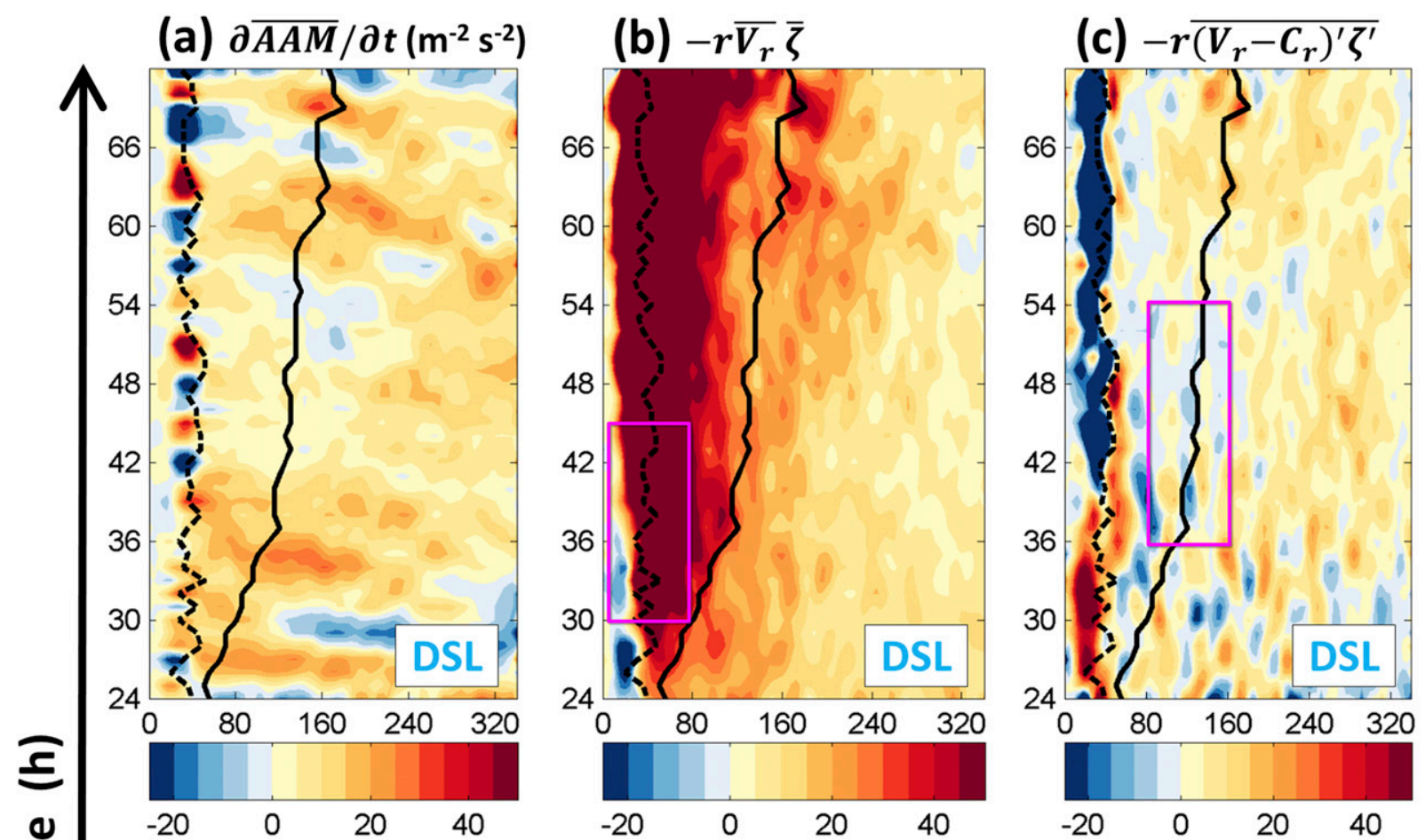

$\stackrel{\text { E }}{\boldsymbol{E}}$
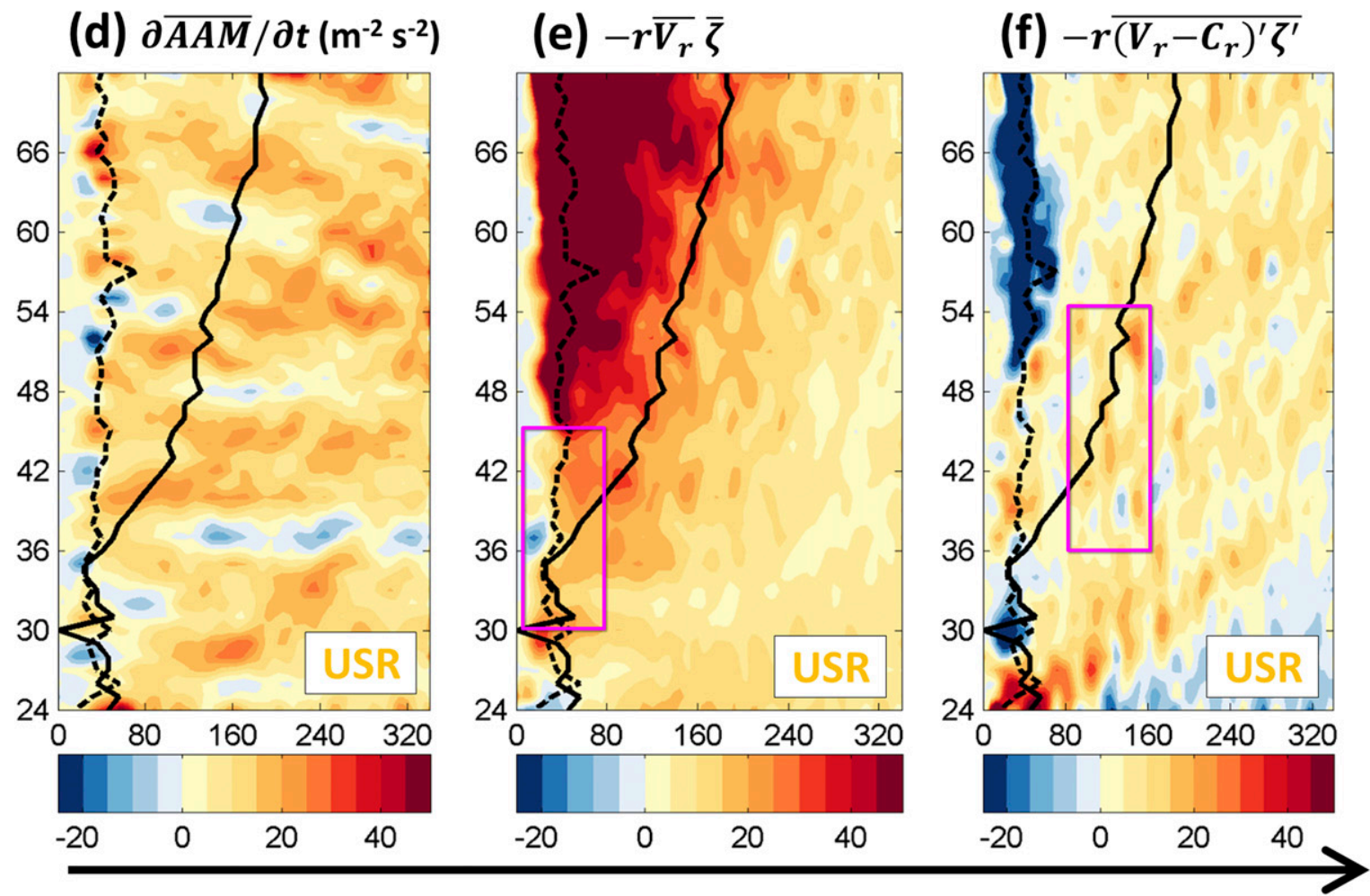

\section{Radius (km)}

FIG. 9. Time-radius Hovmöller diagrams for the TCs in (a)-(c) Exp. DSL and (d)-(f) Exp. USR of azimuthally averaged (a),(d) 0-1-km AAM tendency, (b),(e) 0-1-km mean radial flux of vorticity, and (c),(f) 0-1-km eddy radial flux of vorticity. The unit of shaded color is $\mathrm{m}^{-2} \mathrm{~s}^{-2}$. Black dotted line and black solid line indicate the RMW and R34, respectively. 

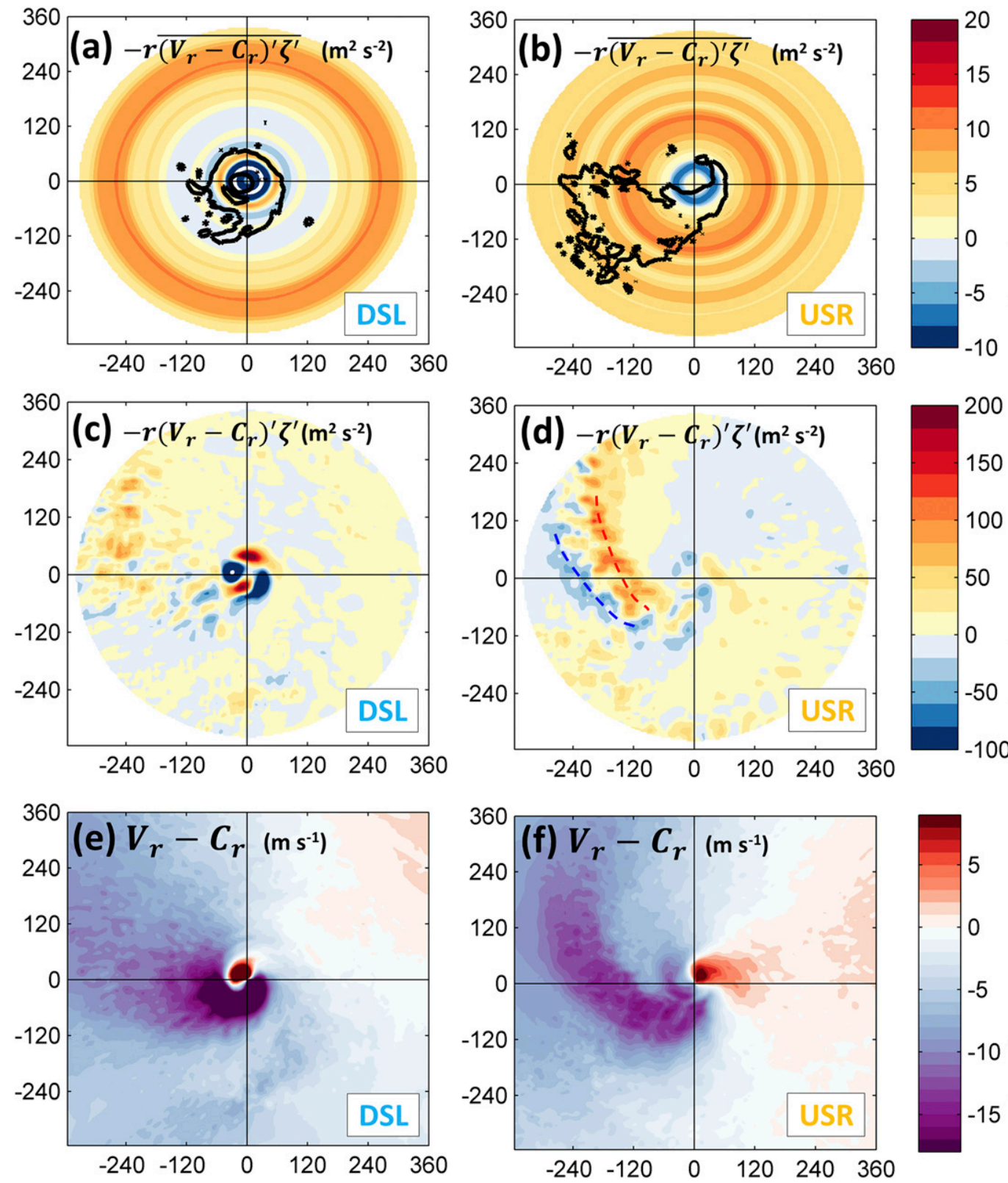

FIG. 10. Selected 42-54-h-averaged fields in the AAM budgets for the TCs in (a),(c),(e) Exp. DSL and (b),(d),(f) Exp. USR: (a),(b) 0-1-km azimuthally averaged eddy radial flux of vorticity and radar reflectivity (black contour at 35 and $50 \mathrm{dBZ}$ ); (c),(d) 0-1-km eddy radial flux of vorticity; and (e),(f) 0-1-km storm-motion-relative radial velocity. All the fields in this figure are smoothed by $20 \mathrm{~km} \times 20 \mathrm{~km}$ areal averaging. The axes are distances $(\mathrm{km})$ from the TC center.

inflow during $42-54 \mathrm{~h}$ are examined for the two experiments (Fig. 10). Whereas a negative azimuthal mean of eddy radial vorticity flux $\left[-r\left(v_{r}-c_{r}\right)^{\prime} \zeta^{\prime}\right]$ is present near the R34 in Exp. DSL (Fig. 10a, approximate 120-km radius), positive $-r \overline{\left(v_{r}-c_{r}\right)^{\prime} \zeta^{\prime}}$ is present near the R34 in Exp. USR (Fig. 10b, approximate $150-\mathrm{km}$ radius). These distinct distributions are attributed to the difference in local eddy radial vorticity flux $\left[-r\left(v_{r}-c_{r}\right)^{\prime} \zeta^{\prime}\right]$ in the downshear rainband regions (Figs. 10c,d). Specifically, the positive $-r\left(v_{r}-c_{r}\right)^{\prime} \zeta^{\prime}$ values near the R34 in Exp. USR (Fig. 10d) are attributed to the active downshear rainbands (Fig. 10b, contour) and the substantial radial inflow associated with these rainbands. Note that Fig. 10d also reveals two banding structures of positive and negative $-r\left(v_{r}-c_{r}\right)^{\prime} \zeta^{\prime}$ values, which may be associated with the updrafts and downdrafts of the organized rainband 
complex (Fig. 10d, gold and blue dashed lines). In summary, it is emphasized that the convection associated with active downshear rainbands is important for TC size expansion by contributing to relatively large eddy radial flux of vorticity near the R34, although this eddy term is generally less important for $\mathrm{TC}$ intensification.

\section{The effect of shear-relative LMF on TC convective features}

As different convective features associated with the distinct LMFs in Exp. DSL and Exp. USR account for the different TC development pathways, this section will explore the processes contributing to the relatively fast eyewall establishment in Exp. DSL (Figs. 11a,c; section 5a) and active and sustained rainbands in Exp. USR (Figs. 11b,d,f; section 5b). This section will also highlight that the simulated TCs modify the background VWS differently in response to the two distinct initially imposed shear-relative LMFs, even though the domain-averaged background shears are generally stable in the simulations (Fig. 1). Specifically, the near-TC VWS in Exp. DSL decreases in magnitude and changes direction substantially after about $36 \mathrm{~h}$ when the eyewall has been established, and the TC has reached a relatively high intensity (Fig. 11c, pink arrow). In contrast, active and sustained rainbands in Exp. USR may be attributed to the near-TC VWS that remains relatively steady (Figs. 11d,f, pink arrow). The evolutions of the local VWS in the two experiments will be further discussed in section $5 \mathrm{~b}$.

\section{a. The fast eyewall establishment in Exp. DSL}

In Exp. DSL, the TC eyewall starts to develop at around $24 \mathrm{~h}$ (Fig. 11a), and the TC exhibits relatively symmetric eyewall convection and less downshear rainband activity at around $36 \mathrm{~h}$ (Fig. 11c). Furthermore, the rainband area in Exp. DSL (Figs. 11a,c) is shifted downwind and radially outward, compared to that in Exp. USR (Figs. 11b,d). Consistent with Riemer et al. (2010), "moisture envelopes" as shown in Fig. 12 with high water vapor mixing ratio within the downshear boundary layer are present in both experiments. Because of the collection of moisture, the air rises (Fig. 12, warm-shaded contours) in the moisture envelope and develops into TC rainbands in the downshear quadrant (see also Figs. 11a,b). Dry air intrudes into the boundary layer from above because of the downward vertical motion on the downstream side of the maximum convection (Fig. 12, cold contours and white shaded color). Note that the moisture envelope in Exp. USR (Figs. 12b,d) is relatively narrow and displaced upstream, compared to that in Exp. DSL (Figs. 12a,c). This displacement between the moisture envelopes accounts for the displacement between the rainbands of the two TCs (Figs. 11a,b).

Although the moisture envelopes are primarily caused by TC-VWS interaction (Riemer et al. 2010), the displacement between the moisture envelopes is further attributed to the LMF-induced surface flux asymmetry (Figs. 13a,b, shading). As the surface latent heat flux is positively correlated with the groundrelative surface wind speed (Figs. 13a,b, shading; Figs. 6b,c, contours), the positive latent heat flux anomaly is present in the downshear-right quadrant in Exp. DSL (Fig. 13a) while in the upshear-left quadrant in Exp. USR (Fig. 13b). To further examine the moisture envelope displacement, backward trajectory analyses (Fig. 13) were applied, and parcels were released at $24 \mathrm{~h}$ and at the lowest model layer in the areas indicated by the purple boxes in Figs. 12c and 12d (corresponding to the boxes in Figs. 13a,b). In each box, twelve parcel trajectories were integrated backward using the Read/Interpolate/Plot 4 (RIP4) software with 5-min time steps. For both TCs, red trajectories entering the left portion of the moisture envelopes come from the northeast or north in the TC outer region, while blue trajectories spiral around the TCs and enter the right portion of the moisture envelopes (Figs. 13a,b). Note that all of these parcels generally stay within the boundary layer (below 1-km height) along their trajectories (not shown).

For Exp. USR (Fig. 13b), air parcels experiencing moderate surface flux $\left(200-400 \mathrm{~W} \mathrm{~m}^{-2}\right)$ enter the right portion of the moisture envelope while air parcels experiencing low surface flux $\left(<200 \mathrm{~W} \mathrm{~m}^{-2}\right)$ enter the left portion of the moisture envelope. These two parcel bundles are suggested to form the narrow and upstreamshifted moisture envelope in Exp. USR (Fig. 12d). For Exp. DSL (Fig. 13a), the red and blue trajectories are accompanied by moderate to high surface flux (200$600 \mathrm{~W} \mathrm{~m}^{-2}$ ) and thus result in the wide and downstreamshifted moisture envelope (Fig. 12c). The evolutions of time-integrated surface flux and mean water vapor mixing ratio for these parcels are also examined (Figs. 13c,d). The red trajectories in Exp. DSL have an averaged boundary layer mixing ratio comparable to that of the blue trajectories at $24 \mathrm{~h}$ (Fig. 13c, red and blue thick lines at $0 \mathrm{~h}$ ). However, in Exp. USR, the red trajectories have an averaged boundary layer mixing ratio lower to that of the blue trajectories (Fig. 13d, red and blue thick lines at $0 \mathrm{~h}$ ). This lower averaged boundary layer mixing ratio is attributed to the relatively low timeintegrated surface latent heat fluxes along the red trajectories (Fig. 13d, thin red lines). 

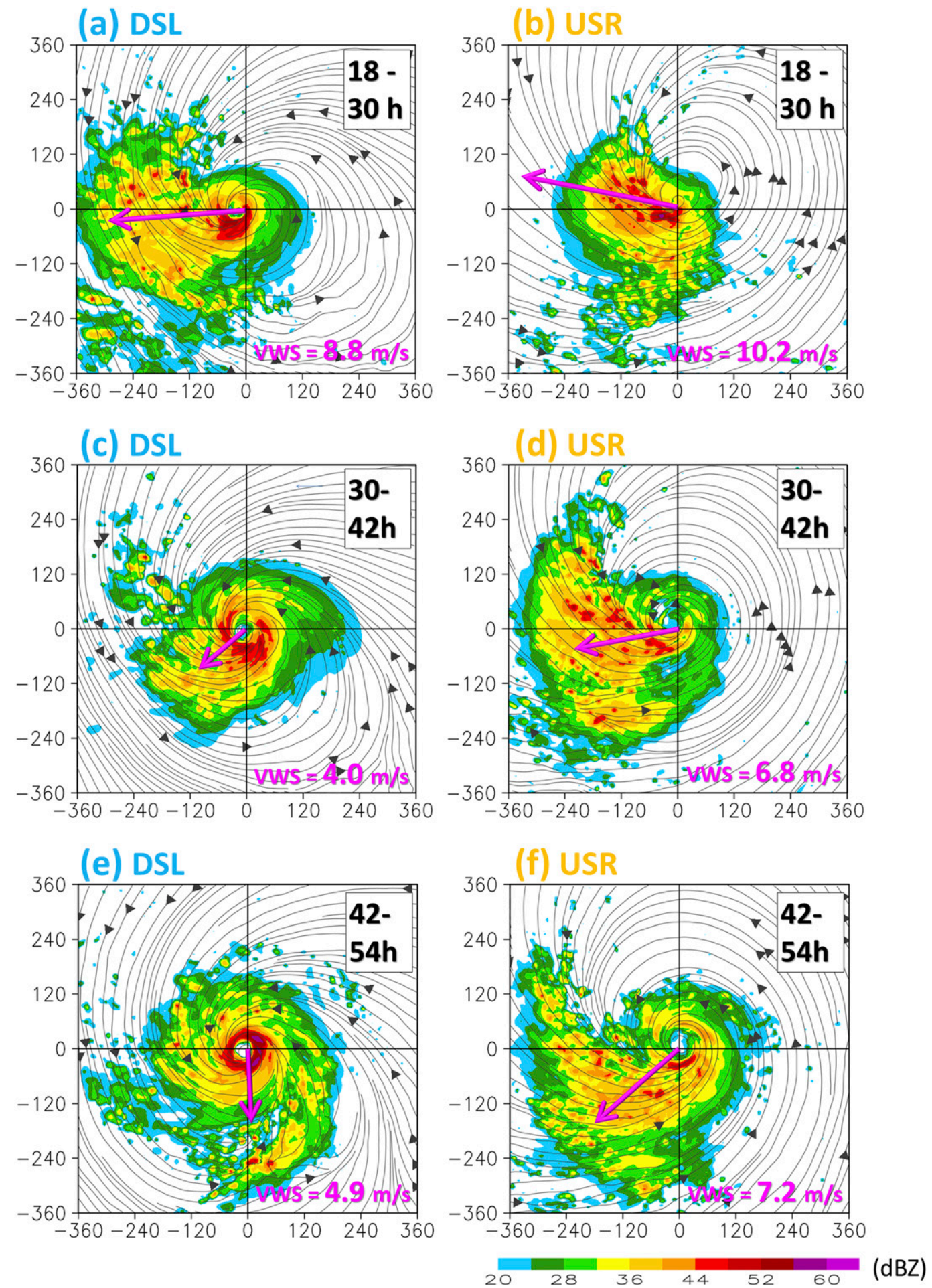

FIG. 11. The 12-h-averaged maximum reflectivity (shaded according to the color bar at lower right) and surface streamlines for the TCs in (a),(c),(e) Exp. DSL and (b),(d),(f) Exp. USR. The time period is labeled at the upper right of each panel. Pink arrow indicates the 12-h-averaged VWS vector within $5^{\circ}$ from the TC center, with the magnitude at the lower right of each panel. The axes are distances $(\mathrm{km})$ from the TC center. 

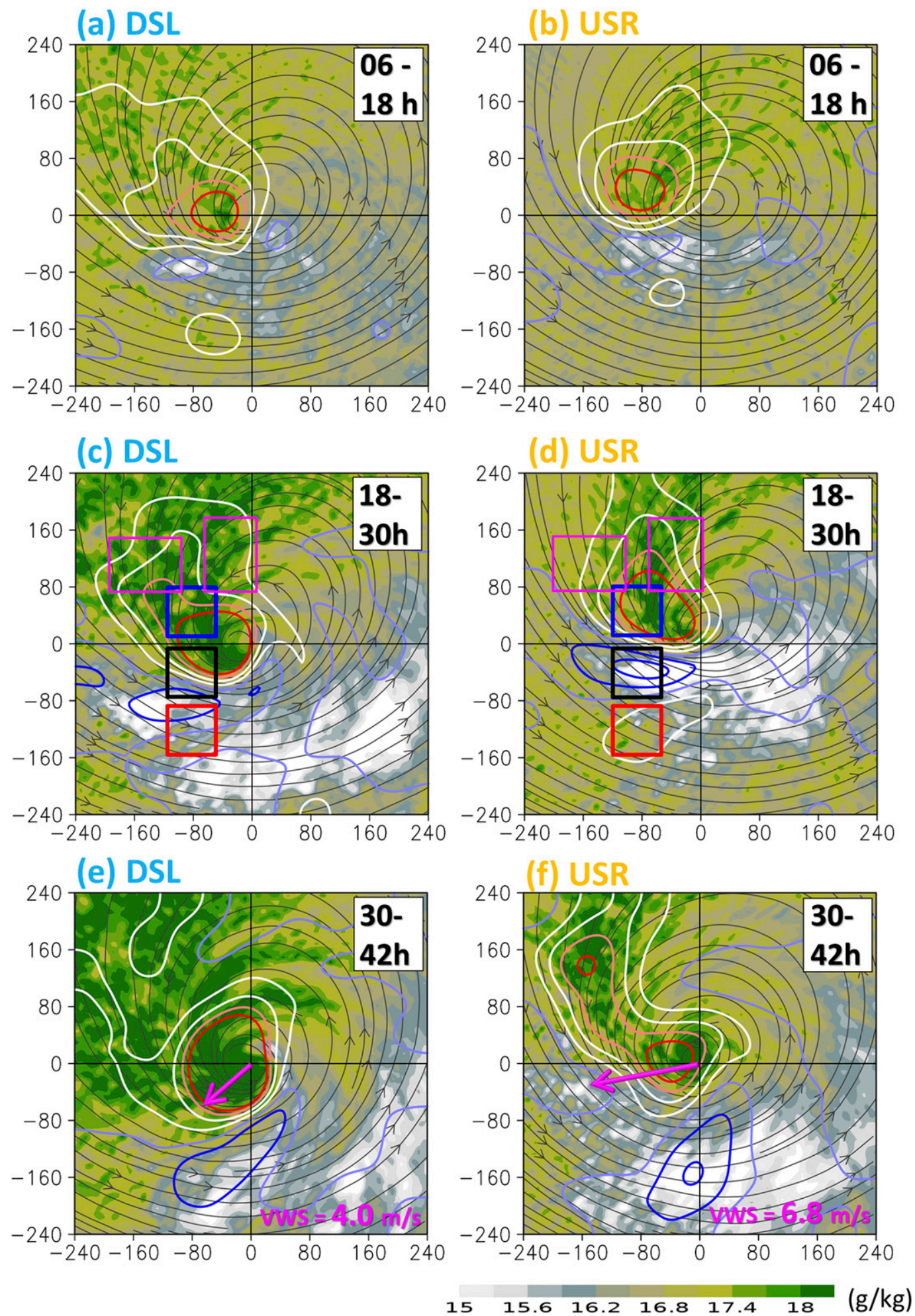

FIG. 12. Selected 12-h-averaged fields for (a),(c),(e) Exp. DSL and (b),(d),(f) Exp. USR at (a),(b) 6-18, (c),(d) 18-30, and (e),(f) 30-42 h. These fields include 0-1-km water vapor mixing ratio (shaded according to the color bar at lower left), $0-1-\mathrm{km}$ vertical velocity [contours, red (blue) contours indicate positive (negative) values at $0.03 \mathrm{~m} \mathrm{~s}^{-1}$ intervals], and $0-1-\mathrm{km}$ streamlines on the TC-motion relative coordinate. Note that the vertical velocity is smoothed by $20 \mathrm{~km} \times$ $20 \mathrm{~km}$ areal averaging. Pink arrows in (e) and (f) indicate the 12-h-averaged VWS vector within $5^{\circ}$ from the TC center, with the magnitude at the lower right of each panel. The axes are distances $(\mathrm{km})$ from the TC center. 
(a) DSL

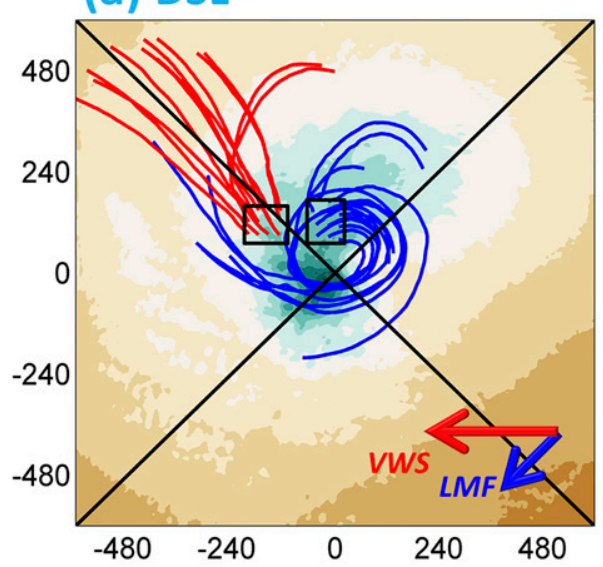

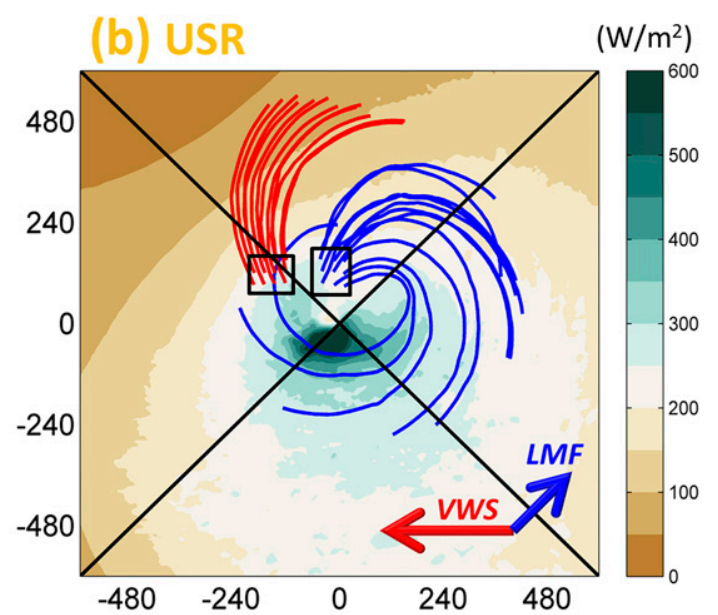

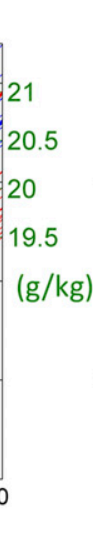

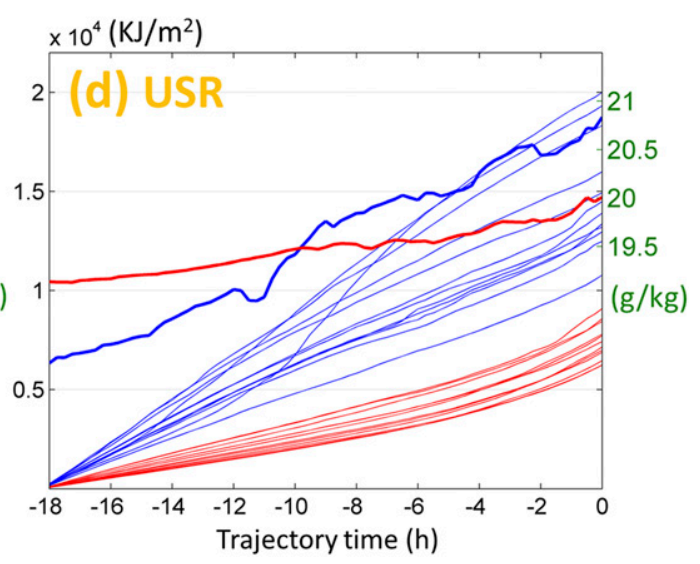

FIG. 13. (a),(b) Averaged surface latent heat flux ( $\mathrm{W} \mathrm{m}^{-2}$, color bar) during 6-24 $\mathrm{h}$ and 18-h backward trajectories released from the black boxes at $0.5-\mathrm{km}$ height at $24 \mathrm{~h}$ (see also the purple boxes in Figs. 12c and 12d) for TCs in (a) Exp. DSL and (b) Exp. USR. The axes are distances ( $\mathrm{km}$ ) from the TC center. (c),(d) Red and blue thick lines are the averaged water vapor mixing ratios ( $\mathrm{g} \mathrm{kg}^{-1}$, right $y$ axis) of the red and blue trajectories in (a) and (b). Red and blue thin lines indicate the time-integrated surface latent heat flux ( $\mathrm{kJ} \mathrm{m}^{-2}$, left $y$ axis) along these trajectories.

In addition to the displacements of moisture envelopes, the storm-motion-relative boundary layer flows also differ between Exp. DSL and Exp. USR (Fig. 12, streamlines; Fig. 14). Figure 14a implies that the downshear-left LMF enhances the boundary layer inflow in the downshear quadrant and suppresses it in the upshear quadrant. However, there is no significant inflow difference above the boundary layer between the two LMF orientations except for the inner-core region (Fig. 14b). A schematic (Fig. 14c) suggests that these storm-motion-relative inflows arise from the friction in the boundary layer. The prescribed LMF vector (Fig. 14c, black arrows), which is equivalent to the prescribed mean flow added to every level, is similar to the TC motion vector caused by the steering effect of the imposed vertically invariant flow. Furthermore, the actual boundary layer mean flow (Fig. 14c, gray dashed line) rotates toward the left-hand side with respect to the
LMF vector because of the friction. Therefore, the TC in Exp. DSL is affected by an eastward storm-motionrelative flow, while the TC in Exp. USR is affected by a westward storm-motion-relative flow (Fig. 14c, red arrows).

This subsection subsequently explores the effect on the fast eyewall establishment in Exp. DSL of both the storm-motion-relative boundary layer flow and surface flux asymmetry (and the moisture envelope displacement) induced by the LMF. Recalling the comparison between Exp. DSL and Exp. USR in Fig. 12, the upward motion (and downward motion) associated with the deep convection in Exp. DSL is shifted downstream and outward (Fig. 12c), and the distribution of the dry air is shifted to the south (Fig. 12c, near the red and black boxes). Recall also that stronger boundary layer inflow is present in the downshear quadrants for Exp. DSL than for Exp. USR (Fig. 14). Subsequently, forward 
a. 0-1-km inflow diff.

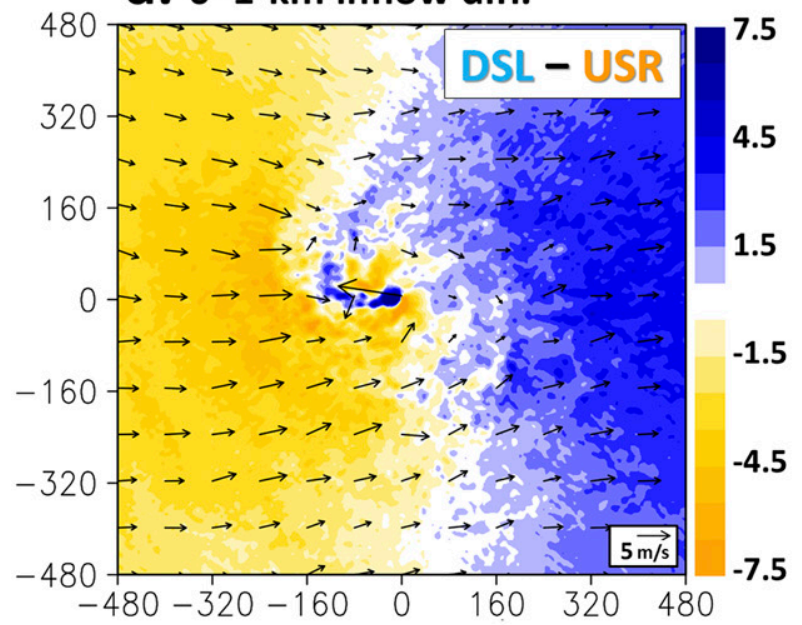

b. 2-4-km inflow diff.
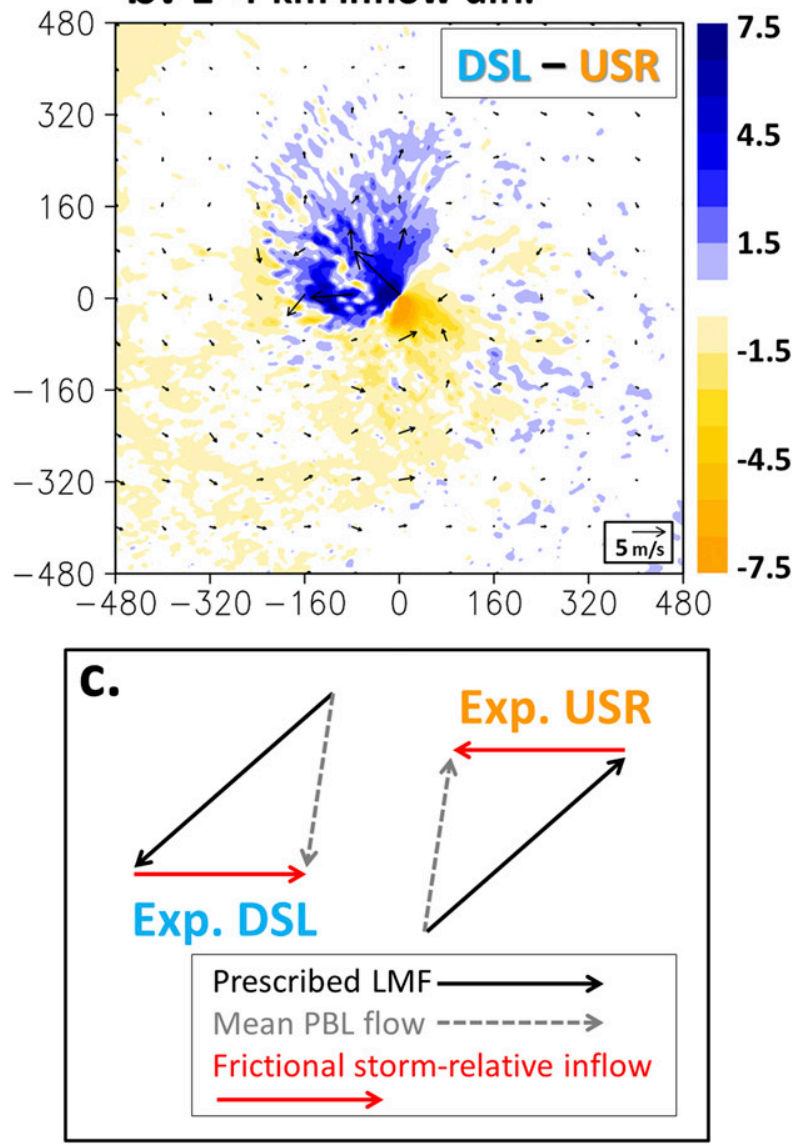

FIG. 14. The difference of storm-motion-relative inflow $\left(\mathrm{m} \mathrm{s}^{-1}\right.$, shaded) and the difference of storm-motion-relative wind $\left(\mathrm{m} \mathrm{s}^{-1}\right.$, vectors scaled at the lower right) between Exp. DSL and Exp. USR at $6-18 \mathrm{~h}$ within (a) 0-1- and (b) 2-4-km height. (c) A schematic showing the prescribed LMFs (black arrows), boundary layer mean flows modified by friction (gray dashed arrows), and frictioninduced storm-motion-relative inflows (red arrows) for the both experiments. trajectories were calculated to examine the moisture transported into the inner core. Parcels were released at $0.5-\mathrm{km}$ height, at $24 \mathrm{~h}$, and from the blue, black, and red boxes as indicated in Figs. 12c and 12d. For Exp. DSL (Fig. 15a), air with high (Fig. 12c, blue box) and moderate (Fig. 12c, black box) moisture flows into the TC inner core, while the dry air in the red box stays away from the inner core. This kind of moisture transport promotes the development of eyewall convection. For Exp. USR (Fig. 15b), the dry air is located within the black box (see also Fig. 12d). Therefore, whereas the air with moderate moisture (Fig. 12d, blue box) flows into the inner core, the dry air (Fig. 12d, black box) wraps around the inner core. Note also that the dry air as shown in Fig. 12d is distributed close to the TC center. As suggested by several studies (e.g., Powell 1990; Riemer et al. 2010), the intrusion of dry air into the TC inner core associated with rainband downdrafts is a critical process to prevent TC intensification. In addition, the evolutions of azimuthally averaged $0-1-\mathrm{km}$ radial moisture fluxes are examined for the two TCs (Fig. 15c) and confirm that the TC in Exp. DSL receives higher moisture fluxes into the TC inner core (Fig. 15c, solid light blue line) than that in Exp. USR (Fig. 15c, solid gold line) during the early development stage (i.e., $12-30 \mathrm{~h}$ ). These higher moisture fluxes may contribute to the well-established eyewall in Exp. DSL at about $30 \mathrm{~h}$. Finally, it is worth noting that the difference in the earlystage intensification between Exp. DSL and Exp. USR could be mainly attributed to the effect of initially imposed shear-relative LMF because the TC circulation has not yet substantially modified the near-TC VWS (see more discussion in the next subsection). However, the intensity evolution afterward in our simulation becomes more complicated because of the modulation of near-TC VWS and is sensitive to the thermodynamic conditions provided (section $3 \mathrm{~b}$ ).

\section{b. The active and sustained rainbands in Exp. USR}

Recall in section 4 that TC size expansion in Exp. USR is attributed to the active and sustained downshear rainbands (Figs. 11d,f), which contribute to large eddy radial fluxes of vorticity near the R34. The actual VWS affecting the TC is examined to explain the rainband development (Fig. 11, pink arrows). The VWS was calculated based on the method described in Galarneau and Davis (2013) and Chen et al. (2018). As applied here, both the rotational and divergent winds associated with a TC vortex have been removed within $5^{\circ}$ from the TC center, and the mean flow at each level is calculated. Then the VWS is defined as the difference between 200and $850-\mathrm{hPa}$ mean flows. Whereas the VWS in Exp. DSL rotates about $90^{\circ}$ and becomes weaker at $42-54 \mathrm{~h}$ 

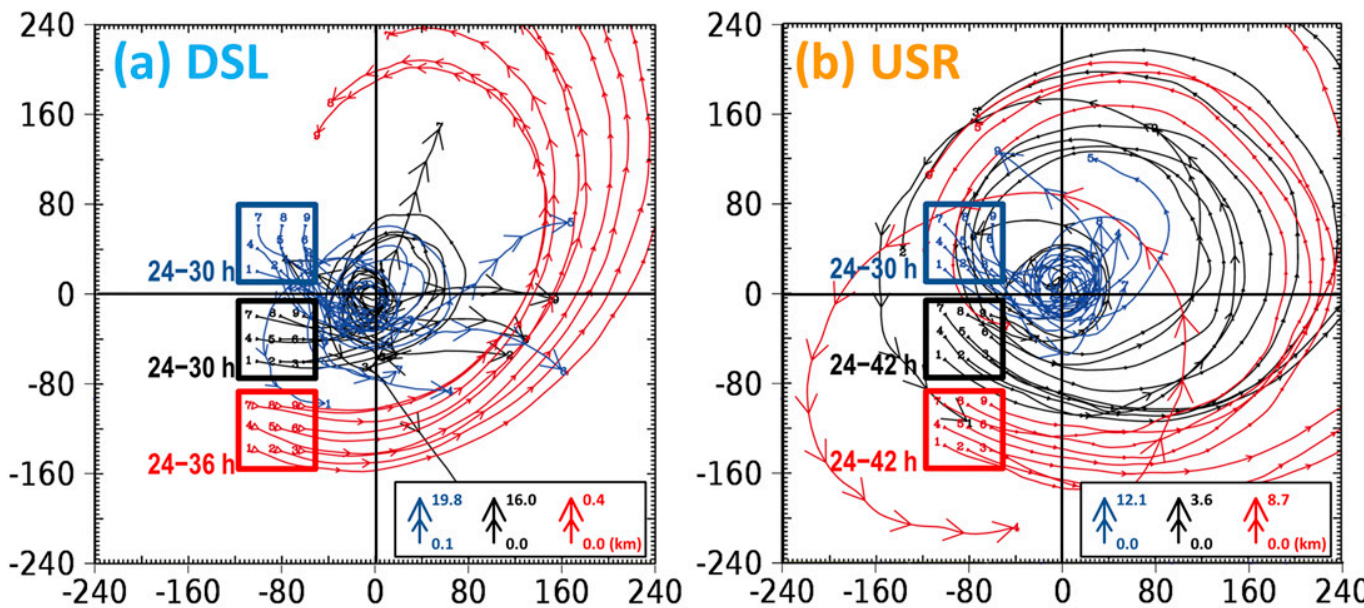

(c) 0-1-km radial inward moisture flux

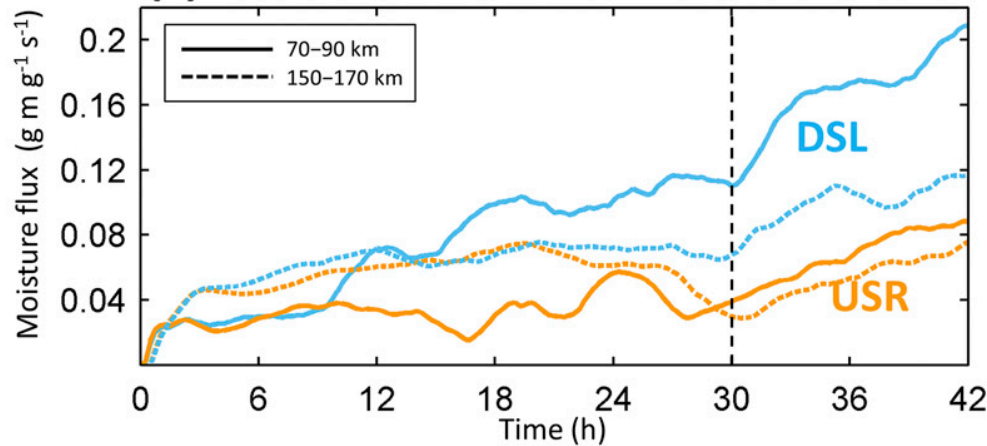

FIG. 15. Forward trajectories released at $0.5-\mathrm{km}$ height at $24 \mathrm{~h}$ in the blue, black, and red boxes (see also the boxes in Figs. 12c and 12d) for the TCs in (a) Exp. DSL and (b) Exp. USR. The colored texts indicate the duration of the trajectories. The size of the arrowheads represents the height of air parcels (see inset at the lower right). The axes are distances $(\mathrm{km})$ from the TC center. (c) Azimuthally averaged 0-1-km radial inward moisture flux $\left[-\left(V_{r}-C_{r}\right) \times q ; \mathrm{g} \mathrm{m} \mathrm{g}^{-1} \mathrm{~s}^{-1}\right]$ at the radii of 70-90 (solid lines) and 150-170 km (dotted lines) for Exp. DSL (light blue) and Exp. USR (gold).

(Fig. 11e, VWS $=4.9 \mathrm{~m} \mathrm{~s}^{-1}$ ), the VWS in Exp. USR still has $72 \%$ of the initial magnitude (Fig. 11f, VWS = $7.2 \mathrm{~m} \mathrm{~s}^{-1}$ ) at $42-54 \mathrm{~h}$. Furthermore, the rainband convection in Exp. DSL becomes weaker after $30 \mathrm{~h}$ (Figs. 11c,e), while the area of convection in Exp. USR becomes more extensive (Figs. 11d,f). The suppression of rainbands in Exp. DSL may be due to the higher TC intensity, which contributes to more subsidence in the rainband region.

On the other hand, consistent with previous observational studies (e.g., Corbosiero and Molinari 2002; Hence and Houze 2011; Reasor et al. 2013), the relatively strong VWS may promote active and sustained rainbands in the downshear quadrants for Exp. USR (Figs. 11d,f). Whereas Exp. USR has an elongated and banding area of strong boundary layer ascent in the downshear-right (northwest) quadrant (Fig. 12f, contours), Exp. DSL has weaker upward motion in the rainband region and concentrated upward motion in the inner core (Fig. 12e, contours). The elongated region of boundary layer ascent in Exp. USR seems to be a crucial aspect that helps force the rainband as the band rotates around the vortex. Moreover, the difference of the boundary layer ascent between the two experiments is suggested to be associated with the difference between the deep-layer VWS vectors (Figs. 12e,f, arrows).

An examination of the east-west cross sections of the vortex structures at $36 \mathrm{~h}$ (Fig. 16) further suggests that the vortex in Exp. USR exhibits a tilted structure with positive vorticity at $6-8 \mathrm{~km}$ extending westward with respect to the surface center (Fig. 16b, contours), while the vortex in Exp. DSL has a vertically aligned structure with a well-developed warm core at $36 \mathrm{~h}$ (Fig. 16a). The westward-extended positive vorticity in the cross section of Exp. USR is related to the horizontal asymmetry in midlevel vorticity and associated with positive and negative temperature anomalies above and below (Fig. 16b, color shading). Several studies have examined the development of secondary circulation in response 
a. West-east cross section

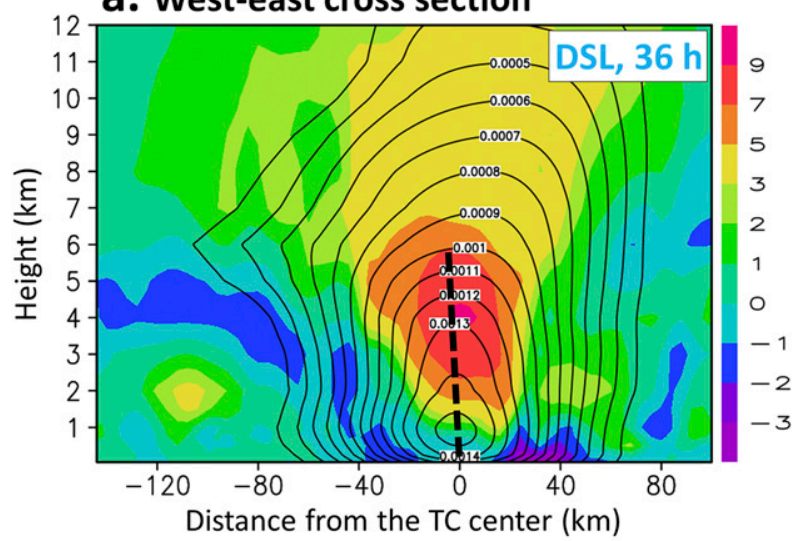

b. West-east cross section

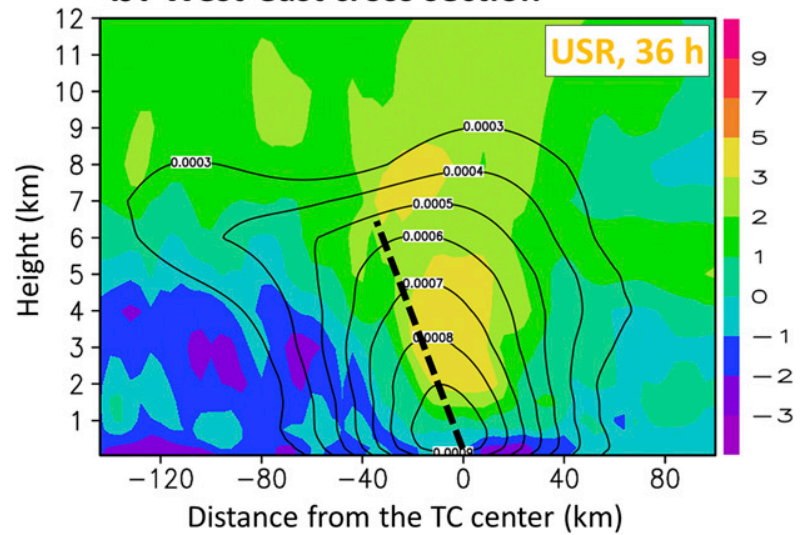

FIG. 16. The $x-z$ cross section of perturbation temperature (shaded, K) and smoothed relative vorticity (contours from 0.0003 to $0.0014 \mathrm{~s}^{-1}$ ), to which nine-point average is applied 50 times, for (a) Exp. DSL and (b) Exp. USR at $36 \mathrm{~h}$. The perturbation temperature is defined as the deviation from the mean temperature within the $500-\mathrm{km}$ radius from the TC center at each level.

to a tilted vortex (e.g., Jones 1995; Frank and Ritchie 2001; Riemer et al. 2010; Riemer 2016). Jones (1995) performed simulations in a dry model and found that the balanced response of the tilting vortex contributed to a tilted isentropic surface that reached its maximum height downtilt. Subsequently, the primary circulation of the vortex followed these tilted isentropic surfaces and dominated the overall vertical motion field, producing the maximum ascent on the downshear-right quadrant. Furthermore, the active rainband development in Exp. USR could be also attributed to the processes documented in Riemer et al. (2010) and Riemer (2016). The tilted TC structure contributed to frictional convergence underneath the positive, low-level vorticity anomaly. This area of frictional convergence extended well outside the eyewall and forced ascent out of the boundary layer, initiating the rainband convection within the moisture envelope.
The evolutions of the near-TC deep-layer VWS are subsequently examined (Fig. 17). Although the actual VWS vector exhibits a counterclockwise rotation (Fig. 11, arrows; Fig. 17a, red arrows), the LMF vector is relatively stable in each experiment (Fig. 17a, blue arrows). The reduction of the VWS magnitude starting from $30 \mathrm{~h}$ in Exp. DSL (reducing 50\% magnitude) is attributed to the early developed eyewall convection (section 5a) and the relatively high intensity compared to that in Exp. USR. Furthermore, the rotations of VWS vectors are attributed to the westward drift of negative vorticity in the upper troposphere (Figs. 17b,c, color shading). For Exp. DSL at $36 \mathrm{~h}$, the upper-level anticyclone is located about $800 \mathrm{~km}$ west to the TC center and contributes to a northwesterly background flow to the TC (Fig. 17b, red vectors in the black circle), thus reducing the VWS magnitude (Fig. 17a, 36h). For Exp. USR, a weaker northwesterly background flow associated with the anticyclone is present (Fig. 17c, red vectors in the black circle). Therefore, the strength difference of upper-level anticyclones between the two experiments (Figs. 17b,c, red vectors) explains the smaller VWS magnitude for Exp. DSL. More detailed analyses regarding the development and evolution of the upperlevel anticyclone, and their modulation on the VWS are left for future investigations.

In summary, for Exp. DSL, the suppressed rainband activity after $36 \mathrm{~h}$ may be partly due to the early intensification of the TC and the reduction of near-TC VWS. Furthermore, the rotation and weakening of the deeplayer VWS constrain the active rainband development (Figs. 11c,e). For Exp. USR, the maintained VWS magnitude accompanied by the elongated area of boundary layer ascent in the downshear-right quadrant (Fig. 12f) due to the tilted vortex structure is critical to the development of active rainbands (Figs. 11d,f). However, in addition to the evolution of deep-layer VWS, it is possible that other mesoscale processes also contribute to the development of active rainbands in Exp. USR, such as the interactions among the convection cells, cold pool, and low-level local vertical shear. But a comprehensive examination of these processes is out of the scope of the current paper and will be presented in our future manuscript.

\section{Conclusions}

This study explores the effects of eight shear-relative LMF orientations on TC intensity and size under initially imposed equivalent deep-layer VWS by analyzing idealized WRF simulations. Note that the depth and height of the imposed VWS (Finocchio et al. 2016) are equivalent among our experiments, and the initial 

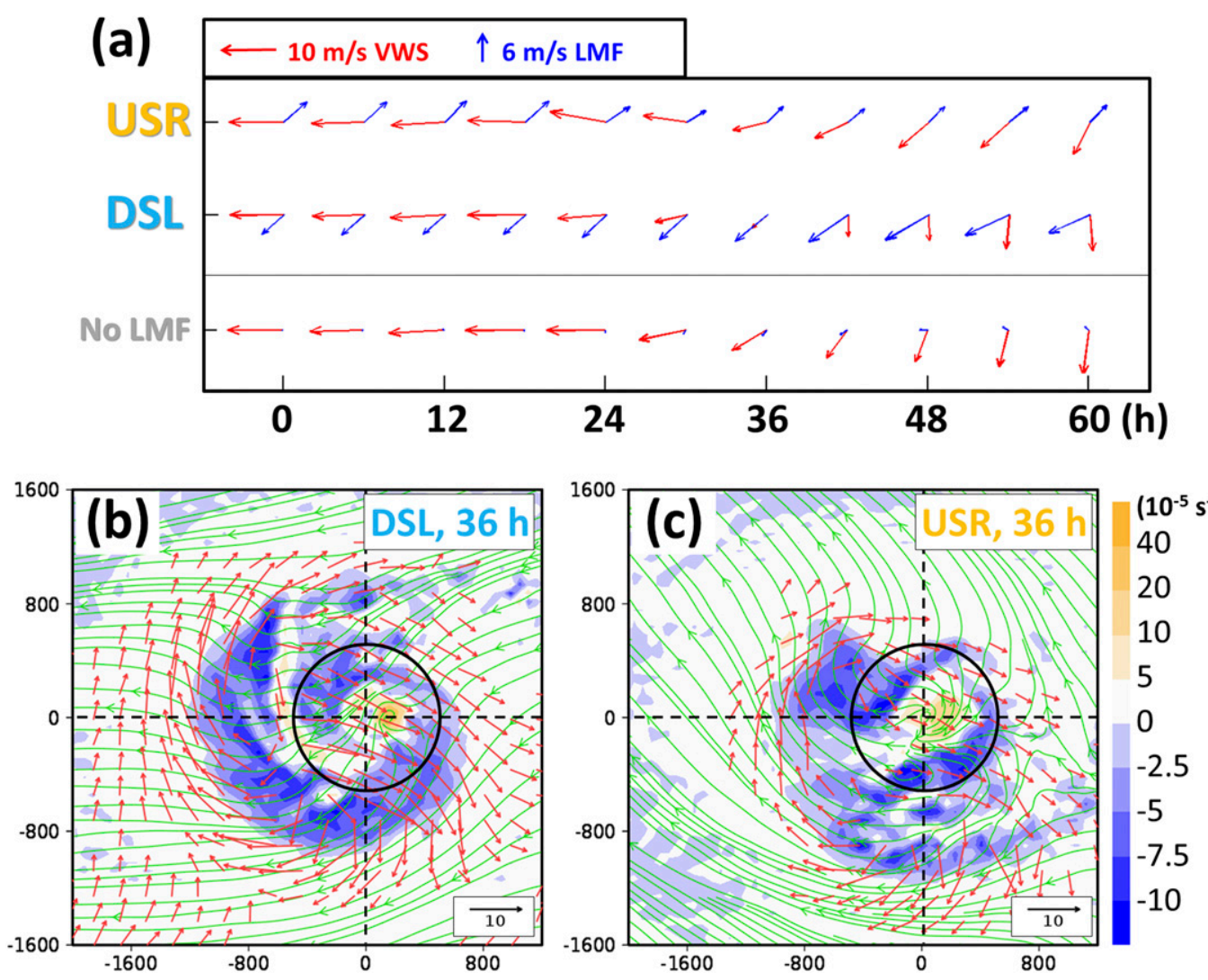

FIG. 17. (a) VWS vectors and LMF vectors every $6 \mathrm{~h}$ from 0 to $60 \mathrm{~h}$ for Exp. USR, Exp. DSL, and the no-LMF experiment. Relative vorticity at $200 \mathrm{hPa}$ [shaded according to the color bar to the right of (c)], 200-hPa stream lines, and 200-hPa wind vectors from which the TC circulation and the prescribed flow (wind at $t=0 \mathrm{~h}$ ) are removed for (b) Exp. DSL and (c) Exp. USR at $36 \mathrm{~h}$. The axes are distances (km) from the TC center. The black circle indicates the $500-\mathrm{km}$ radius from the TC center.

environmental helicities (Onderlinde and Nolan 2014, 2016) for various experiments are small and similar to each other because the $y$ components of the TC-motionrelative LMFs are near zero for all cases. Although the background VWS is stable at a relatively large scale (e.g., the average over the $7200 \mathrm{~km} \times 7200 \mathrm{~km}$ outer domain), our experimental design allows simulated TCs to modify the near-TC VWS differently in response to various LMFs and aims to reproduce the relationship between LMF orientation and TC development as documented by our previous observational study (Chen et al. 2018).

In agreement with Chen et al. (2018), the CTL simulations show that the TC affected by a LMF with an upshear-right orientation favors expansion while a downshear-left LMF is favorable for intensification. The TC interacting with a right-of-shear LMF has the lowest intensification rate. Furthermore, the sensitivity tests of including ocean feedback and increasing SST suggest that $\mathrm{TC}$ intensification in response to various imposed LMF orientations is sensitive to the thermodynamic conditions provided, except for during the early development stage before $36 \mathrm{~h}$. During the first 36-h simulation, TCs affected by downshear-left LMF have high intensification rates in all of the three experiment sets (CTL, higher SST, and ocean feedback). Thus, the analyses for Exp. DSL are focused on the early development stage. On the other hand, TCs affected by upshear-right LMFs have relatively high expansion rates relative to the intensification in all of the three experiment sets.

This paper focuses on the boundary layer processes associated with distinct LMFs and their interaction with the VWS-induced features. These two distinct LMF orientations lead to significant differences in the boundary layer $\theta_{e}$ distribution and TC convective features. For the TC in Exp. DSL, the superposition of the LMF and the TC circulation increases the latent heat fluxes in the downshear outer region while decreases the fluxes in the upshear quadrants. The trajectory analyses suggest that this flux asymmetry contributes to azimuthally downwind and outward displacement of the moisture envelope as well as the rainbands, which are primarily induced by the 
TC-VWS interaction. In addition to the displacements of moisture envelopes, the storm-motion-relative boundary layer flows also differ between the two experiments because of the frictional effect on the boundary layer mean flow. A relatively strong inflow is present in the downshear quadrants for Exp. DSL. Forward trajectory analyses suggest that the fast eyewall establishment in Exp. DSL is attributed to both the storm-motion-relative boundary layer flow and surface flux asymmetry (and the moisture envelope displacement) induced by the LMF. These combined effects lead to higher boundary layer moisture fluxes into the TC inner core and active inner-core convection. Furthermore, the AAM budget confirms that higher mean radial vorticity fluxes in the Exp. DSL are associated with the early developed eyewall. These large mean radial fluxes of vorticity near the RMW contribute to the large intensification rate for the TC in Exp. DSL. In summary, the difference in the early-stage intensification between Exp. DSL and Exp. USR could be mainly attributed to the effect of initially imposed shear-relative LMF because the TC circulation has not yet substantially modified the near-TC VWS. However, after the eyewall establishment in Exp. DSL, the relatively strong and vertically aligned vortex contributes to the reduction of near-TC VWS and thus exhibits a relatively high intensification rate afterward.

Although Rappin and Nolan (2012) also suggested that the asymmetric surface latent heat flux plays a critical role in modifying the axisymmetric TC convection, they emphasized the importance of boundary layer $\theta_{e}$ recovery in the upshear-left quadrant to TC intensification in their simulations with a higher SST. In our CTL simulations with a lower SST, the TC in Exp. US probably lacks effectively high upshear-left surface fluxes and sufficient recovery of boundary layer $\theta_{e}$ associated with the LMF to lead to a higher intensification rate than that in Exp. DS. Therefore, this study alternatively emphasizes the role in TC intensification of both the friction-induced boundary layer inflow and the displacement of the moisture envelope due to the surface flux asymmetry.

The results also show that the near-TC VWS vector in both Exp. DSL and Exp. USR rotates cyclonically because of the downshear-ward drift of the upper-level anticyclone. Moreover, the near-TC VWS in Exp. USR maintains its magnitude while the near-TC VWS in Exp. DSL decreases in magnitude after the eyewall establishment. The maintained VWS magnitude in Exp. USR may promote active and sustained rainbands, while the decreasing VWS magnitude in Exp. DSL may favor a relatively symmetric convection feature. In addition, the AAM budgets confirm that, in Exp. USR, the interaction between the active rainbands and the storm-relative radial inflows in the downshear quadrants promotes TC expansion by increasing the eddy radial flux of vorticity near the R34.

This study emphasizes the role of shear-relative LMF orientation on the structural development of a vertically sheared TC and addresses possible processes accounting for this variability in the real atmosphere (Chen et al. 2018). Future research will continue to explore the sensitivity to thermodynamic profiles and initial vortex structures. The sensitivity to various VWS and LMF magnitudes also needs further investigations. Furthermore, validation of these physical processes by using simulations of historical TCs is a crucial step to understand the effects of shear-relative LMF to TCs in the real atmosphere and to apply these findings for improving operational TC structure forecast.

Acknowledgments. Computing resources and access to the RDA datasets were provided by NCAR's Computational Information Systems Laboratory. This work was funded by the Grant 105-2917-I-564-013 of Ministry of Science and Technology, Taiwan and the National Science Foundation under Cooperative Agreement AGS1033112. The National Center for Atmospheric Research is sponsored by the National Science Foundation.

\section{REFERENCES}

Bhatia, K. T., and D. S. Nolan, 2013: Relating the skill of tropical cyclone intensity forecasts to the synoptic environment. Wea. Forecasting, 28, 961-980, https://doi.org/10.1175/WAF-D-1200110.1.

Chan, K. T., and J. C. Chan, 2014: Impacts of initial vortex size and planetary vorticity on tropical cyclone size. Quart. J. Roy. Meteor. Soc., 140, 2235-2248, https://doi.org/10.1002/qj.2292.

Chen, B.-F., C.-S. Lee, and R. L. Elsberry, 2014a: On tropical cyclone size and intensity changes associated with two types of longlasting rainbands in monsoonal environments. Geophys. Res. Lett., 41, 2575-2581, https://doi.org/10.1002/2014GL059368.

_ R. L. Elsberry, and C.-S. Lee, 2014b: Origin and maintenance of the long-lasting, outer mesoscale convective system in Typhoon Fengshen (2008). Mon. Wea. Rev., 142, 2838-2859, https://doi.org/10.1175/MWR-D-14-00036.1.

_ C.-S. Lee, and R. L. Elsberry, 2016: Synoptic controls of outer mesoscale convective systems in western North Pacific tropical cyclones. Asia-Pac. J. Atmos. Sci., 52, 11-23, https://doi.org/ 10.1007/s13143-015-0085-2.

- C. A. Davis, and Y. Kuo, 2018: Effects of low-level flow orientation and vertical shear on the structure and intensity of tropical cyclones. Mon. Wea. Rev., 146, 2447-2467, https:// doi.org/10.1175/MWR-D-17-0379.1.

Chen, H., and S. G. Gopalakrishnan, 2015: A study on the asymmetric rapid intensification of Hurricane Earl (2010) using the HWRF system. J. Atmos. Sci., 72, 531-550, https://doi.org/ 10.1175/JAS-D-14-0097.1.

Cheung, K. K. W., and R. L. Elsberry, 2002: Tropical cyclone formations over the western North Pacific in the Navy Operational Global Atmospheric Prediction System forecasts. Wea. 
Forecasting, 17, 800-820, https://doi.org/10.1175/1520-0434(2002) $017<0800$ :TCFOTW $>2.0$. CO;2.

Corbosiero, K. L., and J. Molinari, 2002: The effects of vertical wind shear on the distribution of convection in tropical cyclones. Mon. Wea. Rev., 130, 2110-2123, https://doi.org/10.1175/ 1520-0493(2002)130<2110:TEOVWS >2.0.CO;2.

Dare, R. A., and J. L. McBride, 2011: Sea surface temperature response to tropical cyclones. Mon. Wea. Rev., 139, 3798-3808, https://doi.org/10.1175/MWR-D-10-05019.1.

Davis, C. A., and Coauthors, 2008: Prediction of landfalling hurricanes with the Advanced Hurricane WRF Model. Mon. Wea. Rev., 136, 1990-2005, https://doi.org/10.1175/2007MWR2085.1.

DeHart, J. C., R. A. Houze Jr., and R. F. Rogers, 2014: Quadrant distribution of tropical cyclone inner-core kinematics in relation to environmental shear. J. Atmos. Sci., 71, 2713-2732, https://doi.org/10.1175/JAS-D-13-0298.1.

DeMaria, M., and J. Kaplan, 1994: A Statistical Hurricane Intensity Prediction Scheme (SHIPS) for the Atlantic basin. Wea. Forecasting, 9, 209-220, https://doi.org/10.1175/1520-0434(1994) 009<0209:ASHIPS > 2.0.CO;2.

—, and —, 1999: An updated Statistical Hurricane Intensity Prediction Scheme (SHIPS) for the Atlantic and eastern North Pacific basins. Wea. Forecasting, 14, 326-337, https://doi.org/ 10.1175/1520-0434(1999)014<0326:AUSHIP > 2.0.CO;2.

Dunion, J. P., 2011: Rewriting the climatology of the tropical North Atlantic and Caribbean Sea atmosphere. J. Climate, 24, 893 908, https://doi.org/10.1175/2010JCLI3496.1.

- and C. S. Velden, 2004: The impact of the Saharan air layer on Atlantic tropical cyclone activity. Bull. Amer. Meteor. Soc., 85 353-365, https://doi.org/10.1175/BAMS-85-3-353.

Elsberry, R. L., and R. A. Jeffries, 1996: Vertical wind shear influences on tropical cyclone formation and intensification during TCM-92 and TCM-93. Mon. Wea. Rev., 124, 13741387, https://doi.org/10.1175/1520-0493(1996)124<1374: VWSIOT $>2.0 . \mathrm{CO} ; 2$.

Emanuel, K. A., C. DesAutels, C. Holloway, and R. Korty, 2004 Environmental control of tropical cyclone intensity. J. Atmos. Sci., 61, 843-858, https://doi.org/10.1175/1520-0469(2004) 061<0843:ECOTCI >2.0.CO;2.

Finocchio, P. M., S. J. Majumdar, D. S. Nolan, and M. Iskandarani, 2016: Idealized tropical cyclone responses to the height and depth of environmental vertical wind shear. Mon. Wea. Rev., 144, 2155-2175, https://doi.org/10.1175/ MWR-D-15-0320.1.

Fischer, M. S., B. H. Tang, K. L. Corbosiero, and C. M. Rozoff, 2018: Normalized convective characteristics of tropical cyclone rapid intensification events in the North Atlantic and eastern North Pacific. Mon. Wea. Rev., 146, 1133-1155, https:// doi.org/10.1175/MWR-D-17-0239.1.

Frank, W. M., and E. A. Ritchie, 2001: Effects of vertical wind shear on the intensity and structure of numerically simulated hurricanes. Mon. Wea. Rev., 129, 2249-2269, https://doi.org/ 10.1175/1520-0493(2001)129<2249:EOVWSO>2.0.CO;2.

Galarneau, T. J., and C. A. Davis, 2013: Diagnosing forecast errors in tropical cyclone motion. Mon. Wea. Rev., 141, 405-430, https://doi.org/10.1175/MWR-D-12-00071.1.

Hence, D. A., and R. A. Houze, 2011: Vertical structure of hurricane eyewalls as seen by the TRMM Precipitation Radar. J. Atmos. Sci., 68, 1637-1652, https://doi.org/10.1175/2011JAS3578.1.

Hendricks, E. A., M. S. Peng, B. Fu, and T. Li, 2010: Quantifying environmental control on tropical cyclone intensity change. Mon. Wea. Rev., 138, 3243-3271, https://doi.org/10.1175/ 2010MWR3185.1.
Hill, K. A., and G. M. Lackmann, 2009: Influence of environmental humidity on tropical cyclone size. Mon. Wea. Rev., 137, 32943315, https://doi.org/10.1175/2009MWR2679.1.

Hong, S. Y., Y. Noh, and J. Dudhia, 2006: A new vertical diffusion package with an explicit treatment of entrainment processes. Mon. Wea. Rev., 134, 2318-2341, https://doi.org/10.1175/ MWR3199.1.

Jones, S. C., 1995: The evolution of vortices in vertical shear. I: Initially barotropic vortices. Quart. J. Roy. Meteor. Soc., 121, 821-851, https://doi.org/10.1002/qj.49712152406.

Kaplan, J., M. DeMaria, and J. A. Knaff, 2010: A revised tropical cyclone rapid intensification index for the Atlantic and eastern North Pacific basins. Wea. Forecasting, 25, 220-241, https:// doi.org/10.1175/2009WAF2222280.1.

Kepert, J. D., 2006a: Observed boundary-layer wind structure and balance in the hurricane core. Part I. Hurricane Georges. J. Atmos. Sci., 63, 2169-2193, https://doi.org/10.1175/ JAS3745.1.

_ 2006b: Observed boundary-layer wind structure and balance in the hurricane core. Part II. Hurricane Mitch. J. Atmos. Sci., 63, 2194-2211, https://doi.org/10.1175/JAS3746.1.

Lin, I.-I., and Coauthors, 2003: New evidence for enhanced ocean primary production triggered by tropical cyclone. Geophys. Res. Lett., 30, 1718, https://doi.org/10.1029/2003GL017141.

- G. J. Goni, J. A. Knaff, C. Forbes, and M. M. Ali, 2013: Ocean heat content for tropical cyclone intensity forecasting and its impact on storm surge. Nat. Hazards, 66, 1481-1500, https:// doi.org/10.1007/s11069-012-0214-5.

Merrill, R. T., 1984: A comparison of large and small tropical cyclones. Mon. Wea. Rev., 112, 1408-1418, https://doi.org/10.1175/ 1520-0493(1984)112<1408:ACOLAS > 2.0.CO;2.

Montgomery, M. T., and R. K. Smith, 2017: Recent developments in the fluid dynamics of tropical cyclones. Ann. Rev. Fluid Dyn., 49, 541-574, https://doi.org/10.1146/annurevfluid-010816-060022.

, J. A. Zhang, and R. K. Smith, 2014: An analysis of the observed low-level structure of rapidly intensifying and mature Hurricane Earl (2010). Quart. J. Roy. Meteor. Soc., 140, 2132 2146, https://doi.org/10.1002/qj.2283.

Nguyen, L. T., R. Rogers, and P. D. Reasor, 2017: Thermodynamic and kinematic influences on precipitation symmetry in sheared tropical cyclones: Bertha and Cristobal (2014). Mon. Wea. Rev., 145, 4423-4446, https://doi.org/10.1175/MWR-D17-0073.1.

Onderlinde, M. J., and D. S. Nolan, 2014: Environmental helicity and its effects on development and intensification of tropical cyclones. J. Atmos. Sci., 71, 4308-4320, https://doi.org/10.1175/ JAS-D-14-0085.1.

—, and — 2016: Tropical cyclone-relative environmental helicity and the pathways to intensification in shear. J. Atmos. Sci., 73, 869-890, https://doi.org/10.1175/JAS-D-15-0261.1.

Pollard, R. T., P. B. Rhines, and R. Thompson, 1972: The deepening of the wind-mixed layer. Geophys. Fluid Dyn., 4, 381404, https://doi.org/10.1080/03091927208236105.

Powell, M. D., 1990: Boundary layer structure and dynamics in outer hurricane rainbands. Part II: Downdraft modification and mixed layer recovery. Mon. Wea. Rev., 118, 918-938, https://doi.org/ 10.1175/1520-0493(1990)118<0918:BLSADI > 2.0.CO;2.

Rappin, E. D., and D. S. Nolan, 2012: The effects of vertical shear orientation on tropical cyclogenesis. Quart. J. Roy. Meteor. Soc., 138, 1035-1054, https://doi.org/10.1002/qj.977.

Reasor, P. D., and M. T. Montgomery, 2001: Three-dimensional alignment and corotation of weak, TC-like vortices via linear 
vortex Rossby waves. J. Atmos. Sci., 58, 2306-2330, https:// doi.org/10.1175/1520-0469(2001)058<2306:TDAACO >2.0.CO;2.

, — - and L. D. Grasso, 2004: A new look at the problem of tropical cyclones in vertical shear flow: Vortex resiliency. J. Atmos. Sci., 61, 3-22, https://doi.org/10.1175/1520-0469(2004) 061<0003:ANLATP $>2.0 . \mathrm{CO} ; 2$.

, R. Rogers, and S. Lorsolo, 2013: Environmental flow impacts on tropical cyclone structure diagnosed from airborne Doppler radar composites. Mon. Wea. Rev., 141, 2949-2969, https://doi.org/10.1175/MWR-D-12-00334.1.

Riemer, M., 2016: Meso- $\beta$-scale environment for the stationary band complex of vertically sheared tropical cyclones. Quart. J. Roy. Meteor. Soc., 142, 2442-2451, https://doi.org/10.1002/qj.2837.

— clones in vertical wind shear: Lagrangian diagnostic and pathways of environmental interaction. J. Atmos. Sci., 72, 3517-3536, https://doi.org/10.1175/JAS-D-14-0350.1.

— M. T. Montgomery, and M. E. Nicholls, 2010: A new paradigm for intensity modification of tropical cyclones: Thermodynamic impact of vertical wind shear on the inflow layer. Atmos. Chem. Phys., 10, 3163-3188, https://doi.org/10.5194/ acp-10-3163-2010.

,-- , and -2013 : Further examination of the thermodynamic modification of the inflow layer of tropical cyclones by vertical wind shear. Atmos. Chem. Phys., 13, 327-346, https:// doi.org/10.5194/acp-13-327-2013.

Rios-Berrios, R., and R. Torn, 2017: Climatological analysis of tropical cyclone intensity changes under moderate vertical wind shear. Mon. Wea. Rev., 145, 1717-1738, https://doi.org/ 10.1175/MWR-D-16-0350.1.

Sanger, N. T., M. T. Montgomery, R. K. Smith, and M. M. Bell, 2014: An observational study of tropical-cyclone spinup in
Supertyphoon Jangmi from 24 to 27 September. Mon. Wea. Rev., 142, 3-28, https://doi.org/10.1175/MWR-D-12-00306.1.

Schade, L. R., and K. A. Emanuel, 1999: The ocean's effect on the intensity of tropical cyclones: Results from a simple coupled atmosphere-ocean model. J. Atmos. Sci., 56, 642-651, https:// doi.org/10.1175/1520-0469(1999)056<0642:TOSEOT> 2.0.CO;2.

Skamarock, W. C., J. B. Klemp, J. Dudhia, D. O. Gill, D. M. Barker, W. Wang, and J. G. Powers, 2005: A description of the Advanced Research WRF version 2. NCAR Tech. Note NCAR/TN-4681STR, 88 pp., https://doi.org/10.5065/ D6DZ069T.

Smith, R. K., C. W. Schmidt, and M. T. Montgomery, 2011: An investigation of rotational influences on tropical-cyclone size and intensity. Quart. J. Roy. Meteor. Soc., 137, 1841-1855, https://doi.org/10.1002/qj.862.

Stevenson, S. N., K. L. Corbosiero, and J. Molinari, 2014: The convective evolution and rapid intensification of Hurricane Earl (2010). Mon. Wea. Rev., 142, 4364-4380, https://doi.org/ 10.1175/MWR-D-14-00078.1.

Thompson, G., P. R. Field, R. M. Rasmussen, and W. D. Hall, 2008: Explicit forecasts of winter precipitation using an improved bulk microphysics scheme. Part II: Implementation of a new snow parameterization. Mon. Wea. Rev., 136, 5095-5115, https://doi.org/10.1175/2008MWR2387.1.

Wu, C.-C., and K. A. Emanuel, 1993: Interaction of a baroclinic vortex with background shear: Application to hurricane movement. J. Atmos. Sci., 50, 62-76, https://doi.org/10.1175/ 1520-0469(1993)050<0062:IOABVW >2.0.CO;2.

Zhang, F., and D. Tao, 2013: Effects of vertical wind shear on the predictability of tropical cyclones. J. Atmos. Sci., 70, 975-983, https://doi.org/10.1175/JAS-D-12-0133.1. 